\title{
Seemingly injective von Neumann algebras
}

\author{
by \\ Gilles Pisier \\ Texas A\&M University \\ College Station, TX 77843, U. S. A.
}

December 21, 2021

\begin{abstract}
We show that a QWEP von Neumann algebra has the weak* positive approximation property if and only if it is seemingly injective in the following sense: there is a factorization of the identity of $M$

$$
I d_{M}=v u: M \stackrel{u}{\longrightarrow} B(H) \stackrel{v}{\longrightarrow} M
$$

with $u$ normal, unital, positive and $v$ completely contractive. As a corollary, if $M$ has a separable predual, $M$ is isomorphic (as a Banach space) to $B\left(\ell_{2}\right)$. For instance this applies (rather surprisingly) to the von Neumann algebra of any free group. Nevertheless, since $B(H)$ fails the approximation property (due to Szankowski) there are $M$ 's (namely $B(H)^{* *}$ and certain finite examples defined using ultraproducts) that are not seemingly injective. Moreover, for $M$ to be seemingly injective it suffices to have the above factorization of $I d_{M}$ through $B(H)$ with $u, v$ positive (and $u$ still normal).
\end{abstract}

MSC 2010 Classification: 46L10, 46L07, 46B28, 47L07

Keywords: von Neumann algebra, injectivity, positive approximation property 
A von Neumann algebra $M$ is called injective if there exists a Hilbert space $H$ and an isometric normal *-homomorphism $u: M \rightarrow B(H)$ such that there is a projection $P: B(H) \rightarrow u(M)$ onto $u(M)$ with $\|P\|=1$, which by Tomiyama's theorem (see [57]) is automatically a completely positive and completely contractive conditional expectation. Injective von Neumann algebras play a central role in operator algebra theory (see e.g. [57, 58]). Following the work of Connes [19], Choi and Effros [16, 14] proved that a $C^{*}$-algebra $A$ is nuclear if and only if its bidual $A^{* *}$ is injective. Injectivity is in some sense analogous to amenability for groups.

Equivalently $M$ is injective if and only if the identity $I d_{M}$ admits, for some $H$, a factorization of the form

$$
I d_{M}: M \stackrel{u}{\longrightarrow} B(H) \stackrel{v}{\longrightarrow} M
$$

where $u: M \rightarrow B(H)$ and $v: B(H) \rightarrow M$ are completely contractive maps. In fact, it suffices for injectivity to require $u, v$ completely bounded (see Remark 1.4). The fundamental injective example is of course $B(H)$ itself. The main non-injective example is the von Neumann algebra $L(\mathbb{F})$ of a free group $\mathbb{F}$ with at least two generators. Actually, by known results (see [31]) for any embedding $L(\mathbb{F}) \subset B(H)$ as a von Neumann subalgebra there is no bounded linear projection onto $L(\mathbb{F})$. Moreover when $M=L(\mathbb{F})$ there is no factorization as above with $u, v$ both completely bounded (see Remark 1.4). The initial motivation of this note is the observation that when $M=L(\mathbb{F})$, the identity $I d_{M}$ still admits a factorization as above but with $u$ merely a normal unital positive (linearly) isometric embedding and $v$ still completely contractive. When this holds we say that $M$ is "seemingly injective". Actually, for this to hold it suffices to have such a factorization with $u$ and $v$ both positive, and $u$ normal.

In Theorem 1.11, we show that this property characterizes the M's that have a certain form of weak* positive approximation property (in short PAP). The latter was proved for $M=L(\mathbb{F}$ ) in [11] following Haagerup's well known work [28]. Our result can be viewed as analogous to the equivalence between injectivity and semidiscreteness (see [58, p. 173]). In [23, p. 12], Effros and Lance called "semidiscrete" the algebras $M$ that have the weak* completely positive approximation property (in short weak* CPAP). Their paper contains many important fore-runners of Connes's later results on the equivalence of injectivity and semidiscreteness. Connes [19] proved the latter equivalence for factors and Choi and Effros [14] extended it to arbitrary von Neumann algebras. We develop further the parallelism between seemingly injective and injective in $\$ 6$.

\section{Main result}

Throughout this paper, we abbreviate "approximation property" by AP.

We start by introducing the weakening of injectivity, which is the center of interest of this paper:

Definition 1.1. We will say that a von Neumann algebra (or a dual operator space) $M$ is "seemingly injective" if $I d_{M}$ admits, for some $H$, a factorization of the form

$$
I d_{M}: M \stackrel{u}{\longrightarrow} B(H) \stackrel{v}{\longrightarrow} M
$$

with $u$ normal, unital and positive (and hence $\|u\|=1$ ), and $\|v\|_{c b}=1$. Note that $u$ unital and $v u=I d$ implies that $v$ is also unital, and hence completely positive.

Dropping the unital positivity assumption on $u$, if this factorization holds with $u, v$ such that $\|u\|=1$ and $\|v\|_{c b}=1$ we will say that $M$ is remotely injective.

Remark 1.2. In the preceding situation, $u$ is isometric and normal, so by Krein-Smulian the subspace $u(M) \subset B(H)$ is weak* closed, and by Sakai's predual uniqueness theorem (see [55, p. 30]), $u^{-1}: u(M) \rightarrow M$ is weak* continuous. 
Remark 1.3. If $M$ with separable predual is seemingly injective (or remotely injective), we may take $H=\ell_{2}$. Indeed, by the preceding remark $u(M)$ has a separable predual, namely $B(H)_{*} / Z$ where $Z$ is the preanihilator of $u(M)$. It follows that $u(M)$ is normed by a countable subset of the unit ball of $B(H)_{*}$. Therefore there is a separable Hilbert subspace $K \subset H$ such that the compression $\Psi: x \mapsto P_{K} x_{\mid K}$ is an isometric normal unital embedding of $u(M)$ in $B(K)$. Repeating this argument for $M_{n}\left(u(M)\right.$ ) for all $n \geq 1$ (and augmenting $K$ ) we can obtain a separable $K \simeq \ell_{2}$ such that the preceding embedding $\Psi: u(M) \rightarrow B(K)$ is also completely isometric. Then by the injectivity of $B(H)$ the embedding $u(M) \subset B(H)$ factors as $u(M) \stackrel{\Psi}{\longrightarrow} B(K) \stackrel{w}{\longrightarrow} B(H)$ with $w$ completely contractive. Replacing $v$ in (1.1) by $v w$ this shows that we may assume that $H=\ell_{2}$.

Remark 1.4. If $u, v$ are both assumed completely bounded in (0.1) then $M$ is injective (and here we do not need to assume $u$ normal). This is due to Haagerup (see [52, Th. 23.7] together with [52, Cor. 22.19]), refining previous results by Christensen-Sinclair and the author independently showing that the existence of a completely complemented embedding $M \subset B(H)$ (as a von Neumann subalgebra) implies injectivity. See [52, §23.7] for more detailed references. We return to this topic in Remark 4.6 .

Remark 1.5. We could also consider a variant with a constant $c$, and say seemingly $c$-injective if $\|v\|_{c b} \leq c$. There is a natural notion of seemingly nuclear which we do not spell out involving the analogue of the completely positive AP. All this seems to deserve further study.

Remark 1.6. Let $p$ be a projection in $M$. We may view $p M p$ as usual as a von Neumann algebra with unit $p$. It is easy to check that if $M$ is seemingly injective, so is $p M p \oplus \mathbb{C}(1-p)$. Moreover, if $M$ is seemingly injective, so is any von Neumann (unital) subalgebra $N \subset M$ for which there is a unital positive (automatically c.p.) projection $P: M \rightarrow N$ onto $N$. By the last assertion in Theorem 1.11 "unital positive" can be replaced by "positive" in the preceding assertion. In particular $p M p$ is seemingly injective.

Remark 1.7. One major dissemblance with injectivity is that it is unclear (and a priori unlikely to be true) whether the tensor product of two seemingly injective algebras $N, M$ is seemingly injective, even if $N=B(K)$ with $K$ Hilbert. This is of course due to the fact that, unlike for c.p. maps, we cannot tensorize unital positive maps. Analogously, we do not know whether the commutant $M^{\prime}$ is seemingly injective if $M$ is so.

Remark 1.8. Following [42, p. 275], given a constant $c \geq 1$, an operator space $Z \subset B(H)$ is $c$-mixed injective if $Z$ is the range of a projection $P: B(H) \rightarrow Z$ with $\|P\| \leq c$. With this terminology, any remotely injective von Neumann algebra $M$ is isometric to a weak* closed subspace $Z \subset B(H)$ that is 1-mixed injective. Indeed, if (1.1) holds we can take $Z=u(M)$ and $P=u v$.

We will use the weak expectation property (in short WEP) originally introduced by Lance. Recall that a $C^{*}$-algebra $A$ is called WEP (resp. QWEP) if the inclusion $i_{A}: A \rightarrow A^{* *}$ into its bidual factors via completely positive (in short c.p.) and completely contractive (in short c.c.) maps through some $B(H)$ (resp. if it is a quotient of a WEP $C^{*}$-algebra).

Remark 1.9. Kirchberg proved that a von Neumann algebra $M$ is QWEP if and only if there is (for some $H$ ) a u.c.p. map $v: B(H) \rightarrow M$ that is a metric surjection (i.e. $v$ maps the open unit ball of $B(H)$ onto the open unit ball of $M)$. In particular, this shows the following.

Proposition 1.10. A von Neumann algebra $M$ is seemingly injective if and only if there is (for some $H)$ a u.c.p. metric surjection $v: B(H) \rightarrow M$ that admits a normal unital positive lifting $u: M \rightarrow B(H)$.

Our main result is as follows (see $§ 2$ for the definitions on the various forms of AP): 
Theorem 1.11. The following properties of a von Neumann algebra $M$ are equivalent:

(i) $M$ is seemingly injective,

(ii) There is a net of integers $n(\alpha)$ and normal finite rank maps $T_{\alpha}: M \rightarrow M$ of the form

$$
M \stackrel{u_{\alpha}}{\longrightarrow} M_{n(\alpha)} \stackrel{v_{\alpha}}{\longrightarrow} M
$$

such that $u_{\alpha}, v_{\alpha}$ are both unital and positive (so that $\left\|u_{\alpha}\right\| \leq 1,\left\|v_{\alpha}\right\| \leq 1$ ), $u_{\alpha}$ is normal and $T_{\alpha}(x)=v_{\alpha} u_{\alpha}(x) \rightarrow x$ weak $^{*}$ for any $x \in M$ (we then say that $M$ has the matricial weak* PAP).

(iii) $M$ is $Q W E P$ and there is a net $\left(T_{\alpha}\right)$ of normal finite rank unital positive maps on $M$ such that $T_{\alpha}(x) \rightarrow x$ weak $^{*}$ for any $x \in M$ (in which case we say that $M$ has the weak* $\left.P A P\right)$.

Moreover, for $M$ to be seemingly injective it suffices that there is a factorization of the form (1.1) with $u$ normal and $u, v$ both positive.

Remark 1.12. We do not know whether any $M$ with the weak* PAP is QWEP, although the c.p. analogue holds. See [37] for an illuminating discussion on the latter assertion.

Remark 1.13 (On free groups). If $G$ is a free group the metric AP for $C_{\lambda}^{*}(G)$ was discovered by Haagerup [28, as well as the existence of a sequence of completely contractive finite rank multipliers on the von Neumann algebra $L(G)=C_{\lambda}^{*}(G)^{\prime \prime}$ that form a weak* approximation of the identity. Of course the completely positive analogue is excluded since it implies injectivity. Nevertheless, using very similar ideas, Haagerup and de Cannière [11, Th. 4.6] proved that there is a sequence of finite rank normal multipliers that are positive, unital and form a weak* approximation of the identity on $L(G)$ (see also [36] for groups with property $\mathrm{RD}$ with respect to a conditionally negative definite length function). In fact they obtain, for any integer $k \geq 1$ fixed in advance, a sequence of unital $k$-positive multipliers. By (iii) $\Rightarrow$ (i) this shows that $L(G)$ is seemingly injective, and actually we can find a factorization with a $u$ that is $k$-positive, unital and normal.

Remark 1.14 (On Jordan algebras). The ranges of unital positive projections $P$ on $B(H)$ (such as $P=u v$ which projects onto $u(M))$ are described by Effros and Størmer in [25]. They show that such ranges are Jordan algebras for the Jordan product defined by $a \circ b:=P(a b+b a) / 2$. In the situation of Definition 1.1 the map $u: M \rightarrow P(B(H))$ being isometric is a Jordan morphism, and hence satisfies $P(u(x) u(y)+u(y) u(x))=u(x y+y x)$ for all $x, y \in M$.

Perhaps, some version of Theorem 1.11 remains valid when $M$ is only a unital $\mathrm{JBW}^{*}$ algebra, for instance: if such an $M$ is seemingly injective, does it have the weak* metric AP ?

A variant of this question is as follows. Consider a unital weak* closed subspace $Z \subset B(H)$ which is the range of a unital contractive projection. Does $Z$ have the weak* metric AP ? Perhaps one should consider the same question with $B(H)$ replaced by a unital JBW* algebra satisfying some suitable variant of the weak* positive metric AP ?

The range of a contractive projection $P$ on $B(H)$ (or on a $J^{*}$-algebra) is described by Friedman and Russo in [26]. They show that such a range is a Jordan triple system in the triple product $(x, y, z) \mapsto P\left(x y^{*} z+z y^{*} x\right) / 2$. The latter can be faithfully represented as a $J^{*}$-algebra in the sense of Harris [33, 34]. A $J^{*}$-algebra is a norm closed subspace $X \subset B(H, K)(H, K$ Hilbert spaces) that is closed for the operation $X \ni x \mapsto x x^{*} x$. If $u$ and $v$ are contractive in (1.1), $u: M \rightarrow P(B(H))$ is a triple morphism which means that $\left.u\left(x y^{*} z+z y^{*} x\right)=P\left(u(x) u(y)^{*} u(z)+u(z) u(y)^{*} u(x)\right)\right)$ for any $x, y, z \in M$.

Remark 1.15. Let $M$ be seemingly injective as in Definition 1.1 with $u$ normal unital positive and $v$ unital c.p. Then it is easy to check that for any unitary $U$ in $M$, since $\|u(U)\|=1$ and $v(u(U))=U$, $u(U)$ must be in the multiplicative domain of $v$. See e.g. [52, §5.1] or [47] for background on 
multiplicative domains. It follows that $u(M)$ is included in the multiplicative domain of $v$, and hence if $P=u v$, we have

$$
\forall x, y \in M \quad u(x y)=P(u(x) u(y)) \text { and } u(x)^{*}=u\left(x^{*}\right) .
$$

Thus if we equip the range $P(B(H))(=u(M))$ with the product defined by $a \circ b:=P(a b)$ as in [17, Th. 3.1], we find a copy of $M$.

Remark 1.16. The equivalence (i) $\Leftrightarrow$ (ii) in Theorem 1.11 is analogous to the equivalence of injectivity and the weak* CPAP (also often called "semidiscreteness") for von Neumann algebras. This is a celebrated result of Connes [19] and Choi-Effros [14] (see also [62] for an alternate proof). As mentioned by Connes [19, p. 104] part of his argument for injective $\Rightarrow$ semidiscrete is implicit in Effros and Lance's [23, proof of Prop. 4.5]. More details on that kind of argument (that we also use below) can be found at least in the semi-finite case in [52, Th. 8.12, p. 166] (where unfortunately the reduction to the semi-finite case is incorrect).

Remark 1.17 (About "hyperfiniteness"). By Connes's results [19], if $M$ is a finite injective von Neumann algebra there is a net of completely positive normal unital finite rank projections (i.e. idempotent maps) $T_{\alpha}: M \rightarrow M$ that tend pointwise weak* to the identity of $M$. One could wonder whether in the seemingly injective case the same holds with completely positive replaced by positive.

It is known (see [18]) that if we restrict to the non-nuclear and separable predual case, all injective von Neumann algebras are isomorphic as Banach spaces. Actually any infinite dimensional injective operator system on a separable Hilbert space is isomorphic either to $\ell_{\infty}$ or to $B\left(\ell_{2}\right)$ (see [54], and also [9] for a related result). Curiously, the same is true for the free group factors as in Remark 1.13, because of the following fact well known to specialists.

Proposition 1.18. If we restrict to the non-nuclear case, any von Neumann algebra $M$ that is isomorphic (as a Banach space) to a complemented subspace of $B\left(\ell_{2}\right)$ is isomorphic (as a Banach space) to $B\left(\ell_{2}\right)$.

Proof. This follows by a well known application of Pełczyński's decomposition method. Let $\mathscr{B}=$ $B\left(\ell_{2}\right)$ and let $\mathbb{B}$ denote the $\ell_{\infty}$-sense direct sum of the family $\left\{M_{n} \mid n \geq 1\right\}$. On one hand by elementary arguments one shows easily that $\mathscr{B}$ embeds completely isometrically as a complemented subspace in $\mathbb{B}$. On the other hand, by a result due to S. Wassermann ([61] or [52, Th. 12.29]) $\mathbb{B}$ is isometric to a complemented subspace of any non-nuclear $M$. In both cases the projections are contractive, but this is irrelevant at this point. All we need is the following

Fact (Decomposition method): Let $B, M$ be Banach spaces such that each is isomorphic to a complemented subspace of the other. If we also assume $B \simeq \ell_{\infty}(B)$ then $M \simeq B$.

Applying this with $B=\ell_{\infty}(\mathscr{B})$ and $M=\mathscr{B}$, we find $\mathscr{B} \simeq \ell_{\infty}(\mathscr{B})$. Then we can apply this again but with $B=\mathscr{B}$ and $M$ unchanged. This yields the proposition.

We now include the proof of the above fact for the reader's convenience. Assume that $B, M, X, Y$ are Banach spaces such that $B \simeq M \oplus X$ and $M \simeq B \oplus Y$. Since $B \simeq M \oplus X \simeq B \oplus Y \oplus X$, the isomorphism $B \simeq \ell_{\infty}(B)$ implies $B \simeq \ell_{\infty}(B \oplus Y \oplus X)$. By absorption (i.e. since $\mathbb{N} \cup\{0\} \simeq \mathbb{N}$ ), we have

$$
B \oplus Y \simeq \ell_{\infty}(B \oplus Y \oplus X) \oplus Y \simeq \ell_{\infty}(B \oplus Y \oplus X) \simeq B .
$$

Since $M \simeq B \oplus Y$, we conclude $M \simeq B$.

Corollary 1.19. The free group factors $L(G)$ (or the factors described in Remark 1.13) are isomorphic (as Banach spaces) to $B\left(\ell_{2}\right)$. 
Proof. By Theorem 1.11 and Remark 1.3, $L(G)$ is isometric to a complemented subspace of $\mathscr{B}$.

Remark 1.20. The assumption in Proposition 1.18 holds for any QWEP $M$ with separable predual that has the weak* BAP, see Remark 5.8. Indeed, if $M_{*}$ is separable and has the BAP there is a sequence of finite rank normal maps on $M$ tending weak* to the identity.

Our terminology and notation is mostly standard, except that we denote by $M \otimes N$ the algebraic tensor product of two Banach spaces. When $M$ is a von Neumann algebra we also denote by $\bar{M}$ the complex conjugate algebra, i.e. the "same" algebra but with complex conjugate complex multiplication, so that $\bar{M}$ is anti-isomorphic to $M$. For any $y \in M$ we denote by $\bar{y}$ the same element considered as an element of $\bar{M}$. As is well known the mapping $\bar{y} \mapsto y^{*}$ defines an isomorphism from $\bar{M}$ onto the opposite algebra $M^{o p}$ of $M$ (i.e. the "same" algebra but with product in reverse order).

For basic facts and undefined notions, we refer the reader to [57, 58, for operator algebras and to [24, 47, 51] for operator spaces.

\section{Approximation and lifting properties}

A Banach space $X$ has the metric AP (in short MAP) if its identity $I d_{X}$ is the pointwise limit of a net of finite rank contractions. If $X$ is a dual space we say that it has the weak* MAP if there is such a net but converging pointwise to $I d_{X}$ for the weak* topology. Equivalently, assuming $X=\left(X_{*}\right)^{*}$, this means that $X_{*}$ has the MAP. Indeed, by local reflexivity the finite rank contractions on $X$ may be assumed weak* continuous and, taking convex combinations, a pointwise weakly convergent approximating net of finite rank maps on $X_{*}$ can be transformed into a norm convergent one.

An operator system $X \subset B(H)$ has the positive MAP (in short PMAP) if its identity $I d_{X}$ is the pointwise limit of a net of finite rank positive contractive maps. If $X$ is weak* closed in $B(H)$ we say that $X$ has the weak* PMAP if there is such a net formed of weak* continuous maps that converge pointwise to $I d_{X}$ for the weak* topology. In the particular case when $X$ is a von Neumann algebra, the existence of a uniformly bounded net of positive normal finite rank maps tending pointwise weak* to the identity implies the weak* PMAP (see Lemma 5.11). If the net is formed of positive unital maps (as in Definition 2.1) these are contractive so the weak* PMAP is automatic.

To emphasize the parallel with the weak* CPAP of 23 we adopt the following definitions (it would be more precise to add "unital" to weak* PAP and weak* CPAP, but we choose to abbreviate):

Definition 2.1. We say that a von Neumann algebra $M$ has the weak* PAP if there is a net of unital positive normal finite rank maps $\left(T_{\alpha}\right)$ that tend pointwise weak ${ }^{*}$ to the identity on $M$.

We say that $M$ has the matricial weak* PAP if in addition the maps $\left(T_{\alpha}\right)$ admit a matricial factorization as in (ii) in Theorem 1.11 .

We say that $M$ has the weak* CPAP (resp. matricial weak* CPAP) if in addition to the preceding properties the maps $\left(T_{\alpha}\right)$ (resp. $\left.u_{\alpha}, v_{\alpha}\right)$ are all c.p.

Remark 2.2. Let $A$ be a $C^{*}$-algebra with a (self-adjoint 2-sided closed) ideal $I$ so that $A / I$ is a quotient $C^{*}$-algebra. Let $q: A \rightarrow A / I$ be the quotient map. We will denote by $i_{A}: A \rightarrow A^{* *}$ the canonical inclusion. It is well known that the bidual $A^{* *}$ (which is a von Neumann algebra) admits a decomposition as $I^{* *} \oplus\left(A^{* *} / I^{* *}\right)$. In particular, there is a contractive lifting $A^{* *} / I^{* *} \rightarrow A^{* *}$ and a fortiori from $A / I$ to $A^{* *}$. The question whether, when $A / I$ is separable, there is always a bounded (or even an isometric) lifting from $A / I$ to $A$ has remained open since the works of Andersen and 
Ando from the 1970's. In the broader setting of $M$-ideals counterexamples are known (see [32]), but not for ideals in $C^{*}$-algebras. In $\$ 9$ we propose an approach to this question based on the conjecture that there are QWEP von Neumann algebras that are not remotely injective.

We will use several facts due to Andersen [2, Th. 7] (in the real linear setting) and Ando [3] (in full generality), as follows. If $A / I$ has the MAP and is separable there exists a contractive (and hence isometric) lifting $r: A / I \rightarrow A$. More generally, if $X \subset A / I$ is a separable closed subspace with the MAP, the inclusion $X \subset A / I$ admits a contractive lifting $r: X \rightarrow A$. In fact, Ando proved that any map $T: X \rightarrow A / I$ from a separable Banach space $X$ that is the pointwise limit of a net of finite rank contractions from $X$ to $A / I$ admits a contractive lifting $r: X \rightarrow A$, so that $q r=T$. It seems to have remained open ever since Ando's paper [3] whether this holds without the approximability assumption on $A / I$. This problem was studied in Ozawa's PhD thesis [43] (see also [44] and [4]).

If $A / I$ is separable and has the PMAP, T.B. Andersen [2, Th. 7 (3)] proved that there exists a positive isometric lifting $r: A / I \rightarrow A$. Moreover (see [2, Th. 7 (2)]), in the unital case if $X \subset A / I$ is a separable operator system with the PMAP, the inclusion $X \subset A / I$ admits a positive lifting $r: X \rightarrow A$ that is isometric on the self-adjoint elements of $X$. The proof consists in a reduction to the case when $X$ is finite dimensional, which, incidentally, is sketched below in Lemma 2.6. By an elementary argument (see Lemma 2.5), one can obtain a unital positive lifting $r: X \rightarrow A$.

Remark 2.3. The modern way to think of Ando's theorem is through Arveson's principle (see [5. p. 351]) that says that in the separable case pointwise limits of "nicely" liftable maps are "nicely" liftable. More precisely, assume given a separable operator system $X$ and a net of maps $u_{i}: X \rightarrow A / I$ ( $A / I$ being a quotient $C^{*}$-algebra), if each $u_{i}$ admits a lifting in an admissible class (to be defined below) and converges pointwise to a map $u: X \rightarrow A / I$ then $u$ itself admits a lifting in the same class.

A bounded subset $\mathcal{F} \subset B(X, A)$ will be called admissible if for any pair $f, g$ in $\mathcal{F}$ and any $\sigma \geq 0$ in $I_{+}$the mapping

$$
x \mapsto \sigma^{1 / 2} f(x) \sigma^{1 / 2}+(1-\sigma)^{1 / 2} g(x)(1-\sigma)^{1 / 2}
$$

belongs to $\mathcal{F}$. Let $q: A \rightarrow A / I$ denote the quotient map and let

$$
q(\mathcal{F})=\{q f \mid f \in \mathcal{F}\} .
$$

Then Arveson's principle (see [5, p. 351]) says that for the topology of pointwise convergence on $X$ we have

$$
\overline{q(\mathcal{F})}=q(\overline{\mathcal{F}}) .
$$

Actually we do not even need to assume $\mathcal{F}$ bounded if we restrict to the pointwise convergence on a countable subset of $X$.

Remark 2.4. In particular if $X$ is finite dimensional, we do not need to assume $\mathcal{F}$ bounded. For instance, taking for $\mathcal{F}$ the class of positive maps, let $X$ be a finite dimensional operator system. If a map $u: X \rightarrow A / I$ is the pointwise limit of maps that admit positive liftings, then $u$ itself admits a positive lifting.

The classes of positive contractions, unital positive maps, unital c.p. maps, contractions (resp. complete contractions) are all admissible and in the latter case $X$ can be an arbitrary Banach (resp. operator) space. More generally, for each fixed $k \geq 1$, the class of unital $k$-positive maps on an operator system and that of $k$-contractions on an operator space (meaning maps $u$ such that $I d_{M_{k}} \otimes u$ is contractive) are admissible. 
The proof uses quasi-central approximate units in $I$. By this we mean a non decreasing net $\left(\sigma_{\alpha}\right)$ in the unit ball of $I_{+}$such that for any $a$ in $A$ and any $b$ in $\mathcal{I}$

$$
\left\|a \sigma_{\alpha}-\sigma_{\alpha} a\right\| \rightarrow 0 \quad \text { and } \quad\left\|\sigma_{\alpha} b-b\right\| \rightarrow 0
$$

The reasoning is formally the same in all cases as can be checked in the presentations [21, p. 266] or [51, p. 46 and p. 425]. Using this principle, one can reduce the lifting problem of an approximable map $u: X \rightarrow A / I$ roughly to that of lifting finite dimensional subspaces of $A / I$.

The next lemma is a simple well known fact describing how to obtain unital liftings when the map to be lifted is itself unital.

Lemma 2.5. Let $E$ be an operator system and let $u: E \rightarrow A / I$ be a unital positive mapping. If there is a positive lifting $r: E \rightarrow A$ then there is a unital positive one $r^{\prime}: E \rightarrow A$.

Proof. Let $\left(\sigma_{\alpha}\right)$ be a quasi-central approximate unit in $I$. Let $f$ be a state on $E$. Let $r_{\alpha}(x)=$ $f(x) \sigma_{\alpha}+\left(1-\sigma_{\alpha}\right)^{1 / 2} r(x)\left(1-\sigma_{\alpha}\right)^{1 / 2}$. Then $r_{\alpha}(1)-1=\left(1-\sigma_{\alpha}\right)^{1 / 2}[r(1)-1]\left(1-\sigma_{\alpha}\right)^{1 / 2}$ and $r_{\alpha}$ is still a positive lifting. In particular $q\left(r_{\alpha}(1)\right)=1$. Since $r(1)-1 \in I$ we have $\left\|r_{\alpha}(1)-1\right\| \rightarrow 0$ by (2.1). Choosing and fixing $\alpha$ large enough we may assume that $r_{\alpha}(1)$ is invertible. We then set for any $x \in E$

$$
r^{\prime}(x)=r_{\alpha}(1)^{-1 / 2} r_{\alpha}(x) r_{\alpha}(1)^{-1 / 2}
$$

By functional calculus $q\left(r_{\alpha}(1)^{-1 / 2}\right)=q\left(r_{\alpha}(1)\right)^{-1 / 2}=1$. Thus $r^{\prime}$ is a positive unital lifting for $q: A \rightarrow A / I$.

The next Theorem 2.6 is the basic ingredient used in [2] by T.B. Andersen to prove that positive maps admit positive liftings when $E$ is separable with the PMAP.

Theorem 2.6 (Vesterstrøm [60]). Let $E$ be a f.d. operator system and let $u: E \rightarrow A / I$ be a unital positive mapping. Then $u$ admits a unital positive lifting $r: E \rightarrow A$ (i.e. a map such that $q r(x)=u(x)$ for any $x \in E)$.

In [53] Robertson and Smith give a quick direct proof of Theorem 2.6. Moreover, using an averaging argument their proof shows that, for any fixed $n$, Theorem 2.6 remains valid if "positive" is replaced by " $n$-positive" (see also [38] for a different argument).

\section{Standard forms}

We will use the fact (due to Araki, Connes and Haagerup, see [27]) that any von Neumann algebra $M$ admits a "standard form", which means that there is a triple $\left(H, J, P^{\natural}\right)$ consisting of a Hilbert space $H$ such that $M \subset B(H)$ (as a von Neumann algebra), an anti-linear isometric involution $J: H \rightarrow H$ and a cone $P^{\natural} \subset H$ such that

(i) $J M J=M^{\prime}$ and $J x J=x^{*} \quad \forall x \in M \cap M^{\prime}$.

(ii) $P^{\natural} \subset\{\xi \in H \mid J \xi=\xi\}$ and $P^{\natural}$ is self-dual, i.e.

$$
P^{\natural}=\left\{\xi \in H \mid\langle\xi, \eta\rangle \geq 0 \forall \eta \in P^{\natural}\right\} .
$$

(iii) $\forall x \in M \quad J x J x\left(P^{\natural}\right) \subset P^{\natural}$.

(iv) For any $\varphi$ in $M_{*}^{+}$there is a unique $\xi_{\varphi}$ in $P^{\natural}$ such that $\varphi(x)=\left\langle\xi_{\varphi}, x \xi_{\varphi}\right\rangle$ for any $x$ in $M$. 
Remark 3.1. Let $\tau$ be a normal faithful normalized trace on $M$. Then the usual representation $M \rightarrow$ $B\left(L_{2}(\tau)\right.$ ) (of $M$ acting by left multiplication) realizes $M$ in standard form, with $J: L_{2}(\tau) \rightarrow L_{2}(\tau)$ defined by $J(x)=x^{*}$, and $P^{\natural}=L_{2}(\tau)_{+}$. For any $\xi \in L_{2}(\tau)_{+}$and $y, x \in M$ we have $J y J x \xi=x \xi y^{*}$, and if $\xi=1$ we have $\tau\left(x y^{*}\right)=\langle\xi, J y J x \xi\rangle$.

Remark 3.2. Note that when $\varphi$ is a faithful normal state, the unit vector $\xi_{\varphi}$ in $P^{\natural}$ is both separating and cyclic.

Remark 3.3. For any unit vector $\xi \in P^{\natural}$ we have for any $\sum \bar{y}_{j} \otimes x_{j} \in \bar{M} \otimes M$

$$
\left|\sum\left\langle\xi, J y_{j} J x_{j} \xi\right\rangle\right| \leq\left\|\sum \bar{y}_{j} \otimes x_{j}\right\|_{\bar{M}_{\otimes_{\max } M}} .
$$

Indeed, $(\bar{y}, x) \mapsto J y J x$ extends to a (contractive) unital $*$-homomorphism from $\bar{M} \otimes_{\max } M$ to $B(H)$.

In particular, when $M$ is finite with $\tau$ as in Remark 3.1 we have

$$
\left|\sum \tau\left(y_{j}^{*} x_{j}\right)\right| \leq\left\|\sum \bar{y}_{j} \otimes x_{j}\right\|_{\bar{M} \otimes_{\max } M} .
$$

Definition 3.4. Let $A$ be a unital $C^{*}$-algebra. A sesquilinear form $s: A \times A \rightarrow \mathbb{C}$ (equivalent to a linear form on $\bar{A} \otimes A$ ) will be called "bipositive" if it is both positive definite (i.e. such that $s(x, x) \geq 0$ for all $x \in A$ ) and such that

$$
s(a, b) \geq 0 \quad \forall a, b \in A_{+} .
$$

We call it normalized if $s(1,1)=1$.

To any normalized such form $s$ we associate a state $\varphi_{s}$ defined by $\varphi_{s}(x)=s(1, x)$.

Definition 3.5. Let $M$ be a von Neumann algebra. A bipositive (sesquilinear) form $s$ on $M \times M$ such that $x \mapsto s(1, x)$ is a normal state on $M$ is called self-polar if for any $\psi \in M_{+}^{*}$ such that $0 \leq \psi(x) \leq s(1, x)$ for all $x$ in $M_{+}$, there is $a \in M$ with $0 \leq a \leq 1$ such that $\psi(x)=s(a, x)$.

We will use the following basic property of the standard form.

Theorem 3.6. Let $\varphi$ be a faithful normal state on a ( $\sigma$-finite) von Neumann algebra $M$ in standard form. Then the sesquilinear form $s_{\varphi}$ defined for $y, x \in M$ by

$$
s_{\varphi}(y, x)=\left\langle\xi_{\varphi}, J y J x \xi_{\varphi}\right\rangle
$$

is a strictly positive definite self-polar form on $M \times M$ such that

$$
s_{\varphi}(1, x)=\varphi(x) \quad \forall x \in M .
$$

Moreover, a form $\psi \in M_{*}$ satisfies $0 \leq \psi \leq \lambda \varphi(\lambda>0)$ if and only if there is a (uniquely defined) $y \in M$ with $0 \leq y \leq 1$ such that $\psi(x)=\lambda\left\langle\xi_{\varphi}, J y J x \xi_{\varphi}\right\rangle$ for all $x \in M$.

See [52, Th. 23.30 p. 398] for detailed indications of the references.

We make crucial use of the following fact, for which the main idea goes back to Effros and Lance in [23, proof of Prop. 4.5].

Corollary 3.7. Assuming $M$ in standard form, let $\xi \in P^{\natural}$ and let $\varphi \in M_{*}$ be defined by $\varphi(x)=$ $\langle\xi, x \xi\rangle$ for all $x \in M$. Assume that $\varphi \in M_{*}$ is faithful (but not necessarily normalized). Suppose that we have a positive linear map $\psi: \bar{M} \rightarrow M_{*}$ (we could also view $\psi$ as an anti-linear map on $M)$ such that $\psi(\overline{1})=\varphi$. Then there is a unique positive unital linear map $V: M \rightarrow M$ such that

$$
\forall x, y \in M \quad \psi(\bar{y})(x)=\langle\xi, J V(y) J x \xi\rangle .
$$


Proof. We have $0 \leq \psi(\bar{y}) \leq \varphi$ for any $\overline{0} \leq \bar{y} \leq \overline{1}$, and hence by Theorem 3.6 (and an obvious scaling) there is a unique $V(y) \in M$ with $0 \leq V(y) \leq 1$ such that $\psi(\bar{y})(x)=\langle\xi, J V(y) J x \xi\rangle$ for all $x \in M$. Note that $\psi(\overline{1})=\varphi$ guarantees that $V(1)=1$. By the uniqueness of $V(y)$ it is easy to extend $y \mapsto V(y)$ to obtain a unique positive unital linear map $V: M \rightarrow M$ such that

$$
\forall x, y \in M \quad \psi(\bar{y})(x)=\langle\xi, J V(y) J x \xi\rangle .
$$

We will also make use of the following fundamental property of self-polar forms due to Woronowicz and Connes.

Theorem 3.8. Let $s$ be a self-polar form on a ( $\sigma$-finite) von Neumann algebra such that $x \mapsto s(1, x)$ is faithful. Any normalized bipositive form $s^{\prime}$ on $M \times M$ such that $s \leq s^{\prime}$ on the diagonal, must coincide with $s$ on $M \times M$.

See [52, Cor. 23.20] for details.

We will use the following consequence of Theorem 3.8,

Lemma 3.9. For any finite set $\left(x_{j}\right)$ in a von Neumann algebra $M$, we have

$$
\left\|\sum \overline{x_{j}} \otimes x_{j}+\overline{x_{j}^{*}} \otimes x_{j}^{*}\right\|_{\max }=\sup \sum s\left(x_{j}, x_{j}\right)+s\left(x_{j}^{*}, x_{j}^{*}\right),
$$

where the sup runs over all separately normal, normalized, bipositive forms s on $M \times M$, or equivalently over all separately normal bipositive forms $s$ with $s(1,1) \leq 1$.

We refer to [52, (23.15) p. 403] for the proof.

\section{Pietsch-type factorization}

The next statement is a variation on the Pietsch-type factorization for oh-summing maps described in [50]. We say that an embedding $j: B \rightarrow B(\mathcal{H})$ has infinite multiplicity if $\mathcal{H}=H \oplus H \oplus \cdots$ and $j(b)=b \oplus b \oplus \cdots(b \in B)$.

Proposition 4.1. Let $B \subset B(\mathcal{H})$ be an operator space embedded with infinite multiplicity and let $(M, \tau)$ be a tracial probability space. Let us denote by $C_{+}$the set of finite rank operators $h \in B(\mathcal{H})$ such that $h \geq 0$ and $\|h\|_{2}=1$. Let $v: B \rightarrow M$ be a linear map such that $v\left(b^{*}\right)=v(b)^{*}$ for all $b \in B$ (i.e. $v$ is self-adjoint) and for any finite set $\left(b_{j}\right)$ in $B$ such that $t=\sum \overline{b_{j}} \otimes b_{j}$ is self-adjoint we have

$$
\left\|\sum \overline{v\left(b_{j}\right)} \otimes v\left(b_{j}\right)\right\|_{\bar{M} \otimes_{\max } M} \leq\left\|\sum \overline{b_{j}} \otimes b_{j}\right\|_{\bar{B} \otimes_{\min } B}
$$

Then there is a net $\left(h_{\alpha}\right)$ in $C_{+}$such that for any $b \in B$ the following limit exists and we have

$$
\tau\left(v(b)^{*} v(b)\right) \leq \lim \operatorname{tr}\left(h_{\alpha} b^{*} h_{\alpha} b\right) .
$$

Proof. Since $v$ is self-adjoint and $\tau$ tracial, we have $\tau\left(v(b)^{*} v(b)\right)=\tau\left(v\left(b^{*}\right)^{*} v\left(b^{*}\right)\right)$. Then for any self-adjoint $t=\sum \overline{b_{j}} \otimes b_{j}$

$$
\sum \tau\left(v\left(b_{j}\right)^{*} v\left(b_{j}\right)\right) \leq(1 / 2)\left\|\sum \overline{v\left(b_{j}\right)} \otimes v\left(b_{j}\right)+\overline{v\left(b_{j}^{*}\right)} \otimes v\left(b_{j}^{*}\right)\right\|_{\max } \leq(1 / 2)\left\|\sum \overline{b_{j}} \otimes b_{j}+\overline{b_{j}^{*}} \otimes b_{j}^{*}\right\|_{\min }
$$




$$
=\sup _{h \in C_{+}}(1 / 2) \sum \operatorname{tr}\left(h b_{j}^{*} h b_{j}\right)+\operatorname{tr}\left(h b_{j} h b_{j}^{*}\right)=\sup _{h \in C_{+}} \sum \operatorname{tr}\left(h b_{j}^{*} h b_{j}\right)
$$

where for the next to last equality we refer to [52, Lemma 22.22] using the fact that $\|t\|_{\min }=\|t\|_{\max }$ for any $t=\sum \overline{b_{j}} \otimes b_{j} \in \overline{B(\mathcal{H})} \otimes B(\mathcal{H})$. We expand on the latter fact in Remark 4.4.

By a well known variant of Hahn-Banach (see e.g. [52, Lemma A16]) there is a net $\left(\mu_{\alpha}\right)$ of finitely supported probability measures on $C_{+}$such that for any $b \in B$ the following limit exist and we have

$$
\tau\left(v(b)^{*} v(b)\right) \leq \lim \int \operatorname{tr}\left(h b^{*} h b\right) d \mu_{\alpha}(h)
$$

Then using the fact that $B$ is represented on $\mathcal{H}$ with infinite multiplicity one can obtain a net for which (4.7) holds (see e.g. [52, Prop. 4.23] for details).

Remark 4.2. Let $B, M$ be $C^{*}$-algebras and let $v: B \rightarrow M$ be a c.b. (resp. c.p.) map. Then, for any $C^{*}$-algebra $A$, the map $I d_{A} \otimes v$ extends to a bounded map from $A \otimes_{\min } B$ to $A \otimes_{\min } M$ (resp. from $A \otimes_{\max } B$ to $A \otimes_{\max } M$ with norm at most $\|v\|_{c b}$ (resp. $\left.\|v\|\right)$. See e.g. [52, §7]. It follows that the map $\bar{v} \otimes v$ extends to a bounded map from $\bar{B} \otimes_{\min } B$ to $\bar{M} \otimes_{\min } M$ (resp. from $\bar{B} \otimes_{\max } B$ to $\bar{M} \otimes_{\max } M$ with norm at most $\|v\|_{c b}^{2}$ (resp. $\|v\|^{2}$ ).

The next fact, which plays an important role in our paper, seems to have passed unnoticed.

Lemma 4.3. Let $v: B \rightarrow D$ be a positive map between $C^{*}$-algebras. Then for any self-adjoint tensor $t=\sum \overline{x_{j}} \otimes x_{j} \in \bar{B} \otimes B$, we have

$$
\left\|\sum \overline{v\left(x_{j}\right)} \otimes v\left(x_{j}\right)\right\|_{\bar{D} \otimes_{\max } D} \leq\|v\|^{2}\left\|\sum \overline{x_{j}} \otimes x_{j}\right\|_{\bar{B} \otimes_{\max } B}
$$

Proof. The map $v^{* *}: B^{* *} \rightarrow D^{* *}$ is a normal positive map. We have $1 \leq v^{* *}(1) \leq\left\|v^{* *}\right\| 1=\|v\| 1$. Therefore for any separately normal, bipositive form $s: D^{* *} \times D^{* *}$ the composition $s\left(v^{* *}, v^{* *}\right)$ is a form with the same properties. By bipositivity we have $s\left(v^{* *}(1), v^{* *}(1)\right) \leq s(1,1)\|v\|^{2}$. By (3.3) (and by homomogeneity) for any self-adjoint tensor $t=\sum \overline{x_{j}} \otimes x_{j} \in \overline{B^{* *}} \otimes B^{* *}$ we have

$$
\left\|\sum \overline{v^{* *}\left(x_{j}\right)} \otimes v^{* *}\left(x_{j}\right)\right\|_{\overline{D^{* *}} \otimes_{\max } D^{* *}} \leq\|v\|^{2}\left\|\sum \overline{x_{j}} \otimes x_{j}\right\|_{\overline{B^{* *}} \otimes_{\max } B^{* *}} .
$$

But since we have a canonical isometric embedding $\bar{D} \otimes_{\max } D \subset \overline{D^{* *}} \otimes_{\max } D^{* *}$ (and similarly for $B$ ) we obtain (4.3).

Remark 4.4. When $B$ has the WEP, in particular when $B=B(H)$, for any $\left(b_{j}\right)$ in $B$ we have

$$
\left\|\sum \overline{b_{j}} \otimes b_{j}\right\|_{\min }=\left\|\sum \overline{b_{j}} \otimes b_{j}\right\|_{\max } .
$$

See [52, Cor. 22.16] for a detailed proof. Thus when $B=B(H)$ (or when $B$ has the WEP) (4.3) becomes for any self-adjoint $\sum \overline{x_{j}} \otimes x_{j} \in \bar{B} \otimes B$

$$
\left\|\sum \overline{v\left(x_{j}\right)} \otimes v\left(x_{j}\right)\right\|_{\bar{D} \otimes_{\max } D} \leq\|v\|^{2}\left\|\sum \overline{x_{j}} \otimes x_{j}\right\|_{\bar{B} \otimes_{\min } B} .
$$

Moreover, if $v$ is c.p. with $\|v\| \leq 1$ then (4.5) holds for any $\left(x_{j}\right)$ in $B$ by Remark 4.2.

Remark 4.5. Let $R$ and $C$ denote as usual the row and column operator spaces. It is known (see [52, Rem. 22.20]) that if $v: B \rightarrow D$ is such that $\left\|I d_{X} \otimes v: X \otimes_{\min } B \rightarrow X \otimes_{\min } D\right\| \leq 1$ both for $X=R$ and $X=C$ then $v$ satisfies (4.3). Therefore, by Choi's generalization of Kadison's inequality ([12, Cor. 2.8]), any 2-positive contraction satisfies (4.3). Given this, the preceding lemma is not so surprising. In particular, any complete contraction $v$ satisfies (4.3). See [52, Cor. 22.19] for a detailed proof. 
Remark 4.6. Let $u, v$ be such that $I d_{M}=v u$ (as in (1.1) ). Assume that there are constants $c_{u}$ and $c_{v}$ such that for any $n$ and $\left(x_{j}\right)_{1 \leq j \leq n}$ in $M$ and any $\left(b_{j}\right)_{1 \leq j \leq n}$ in $B(H)$ with $\sum \overline{x_{j}} \otimes x_{j}$ and $\sum \overline{b_{j}} \otimes b_{j}$ self-adjoint, we have

$$
\begin{gathered}
\left\|\sum \overline{u\left(x_{j}\right)} \otimes u\left(x_{j}\right)\right\|_{\overline{B(H)} \otimes_{\min } B(H)} \leq c_{u}\left\|\sum \overline{x_{j}} \otimes x_{j}\right\|_{\bar{M} \otimes_{\min } M} . \\
\left\|\sum \overline{v\left(b_{j}\right)} \otimes v\left(b_{j}\right)\right\|_{\bar{M} \otimes_{\max } M} \leq c_{v}\left\|\sum \overline{b_{j}} \otimes b_{j}\right\|_{\overline{B(H)} \otimes_{\max } B(H)} .
\end{gathered}
$$

Then $M$ is injective. Indeed, by (4.4) we have

$$
\left\|\sum \overline{x_{j}} \otimes x_{j}\right\|_{\bar{M} \otimes_{\max } M} \leq c_{u} c_{v}\left\|\sum \overline{x_{j}} \otimes x_{j}\right\|_{\bar{M} \otimes_{\min } M},
$$

which by a theorem due to Haagerup (see [52, Th. 23.7]) implies that $M$ has the WEP and hence (being a von Neumann algebra) that $M$ is injective.

In particular this shows that if $I d_{M}=v u$ with $u$ c.b. and $v$ c.p. then (4.6) and (4.7) hold (see Remark 4.2) and hence $M$ is injective. Actually, much less suffices, as we now explain.

Let $X$ be an operator space, for example $R$ or $C$. Let us say that $v: B \rightarrow D$ is $X$-bounded if $\left\|I d_{X} \otimes v: X \otimes_{\min } B \rightarrow X \otimes_{\min } D\right\|<\infty$. It is known (see [52, p. 375, Rem. 22.20]) that if $v$ is $X$-bounded both for $X=R$ and $X=C$, or equivalently for $X=R \oplus C$, then $v$ satisfies (4.7) (the contractive case is discussed in Remark 4.5).

As for (4.6), assuming $M \subset B(\mathcal{H})$ completely isometrically, it suffices for the same reason that $u$ admits an extension $\widetilde{u}: B(\mathcal{H}) \rightarrow B(H)$ that is $X$-bounded both for $X=R$ and $X=C$. Actually, it suffices that $\widetilde{u}$ be $X$-bounded when $X$ is the operator Hilbert space $O H$ (see [51, p. 122]).

This leads to what seems to be an interesting example. Let $M$ be a non injective von Neumann algebra. Let $u, v$ be maps such that $I d_{M}=v u$ (as in (1.1)). Assume that $u: M \rightarrow B(H)$ is 2-positive and that $v$ satisfies (4.7) (as is the case for e.g. $M=L\left(\mathbb{F}_{\infty}\right)$ ). Then $u: M \rightarrow B(H)$ is an example of a map that is $X$-bounded for $X=R \oplus C$ but does not extend to a similarly bounded $\operatorname{map} \widetilde{u}: B(\mathcal{H}) \rightarrow B(H)$.

\section{Proof of main result}

We start by some simple preliminary elementary propositions.

Proposition 5.1. Let $T: M \rightarrow N$ be a linear map between von Neumann algebras. Assume that there is a net of pairs of positive contractions $u_{\alpha}: M \rightarrow B\left(H_{\alpha}\right), v_{\alpha}: B\left(H_{\alpha}\right) \rightarrow N$, with $u_{\alpha}$ normal, such that $v_{\alpha} u_{\alpha} \rightarrow T$ pointwise-weak*. Then there is (for some $H$ ) a pair of positive contractions $u: M \rightarrow B(H), v: B(H) \rightarrow N$, with u normal, such that $v u=T$. The construction is such that if the $u_{\alpha}$ 's and $v_{\alpha}$ 's are all unital then so are $u, v$, and if the $v_{\alpha}$ 's are all c.p. then so is $v$.

Proof. Let $B=\oplus_{\alpha} B\left(H_{\alpha}\right)$. Let $u: M \rightarrow B$ be defined by $u(x)=\oplus_{\alpha} u_{\alpha}(x)(x \in M)$. Let $p_{\alpha}: B \rightarrow B\left(H_{\alpha}\right)$ be the $\alpha$-th coordinate mapping. We define $v: B \rightarrow N$ by setting $v(b)=$ weak $\lim _{\mathcal{U}} v_{\alpha} p_{\alpha}(b)=$ weak $^{*} \lim _{\mathcal{U}} v_{\alpha}\left(b_{\alpha}\right)$ for any $b=\left(b_{\alpha}\right) \in B$, where $\mathcal{U}$ is an ultrafilter refining the net. Clearly, $v u=T$ and all the announced properties hold. Lastly, as the identity of $B$ factors through $B(H)$ with $H=\oplus_{\alpha} H_{\alpha}$ via unital c.p. normal maps, we may replace $B$ by $B(H)$.

The next lemma is elementary. 
Lemma 5.2. Let $u: M \rightarrow B(H)$ and $v: B(H) \rightarrow M$ be positive maps (resp. with u normal). Let $\theta=v u: M \rightarrow M$. Let $\varepsilon>0$ and $0 \leq \delta<1$. If $\|\theta(1)-1\| \leq \delta$, there are unital positive maps $u^{\prime}: M \rightarrow B(H)$ and $v^{\prime}: B(H) \rightarrow M$ (resp. with $u^{\prime}$ normal) such that

$$
\left\|\theta-v^{\prime} u^{\prime}\right\| \leq\left(2^{1 / 2}+1\right)(\delta+\varepsilon\|v\|)^{1 / 2}+\varepsilon\|v\| .
$$

Moreover, if in addition $v$ is c.p. we obtain $v^{\prime}$ also c.p.

Proof. Let $f \in M_{*}$ be a normal state. Then let $u_{\varepsilon}(x)=u(x)+\varepsilon f(x) 1_{B(H)}$ and let $u^{\prime}(x)=$ $u_{\varepsilon}(1)^{-1 / 2} u_{\varepsilon}(x) u_{\varepsilon}(1)^{-1 / 2}$, and $v_{\varepsilon}(b)=v\left(u_{\varepsilon}(1)^{1 / 2} b u_{\varepsilon}(1)^{1 / 2}\right)$ for $x \in M$ and $b \in B(H)$. Note

$$
v_{\varepsilon} u^{\prime}=v u_{\varepsilon}=\theta+\varepsilon f(\cdot) v(1) .
$$

Then $u^{\prime}: M \rightarrow B(H)$ is unital positive and normal, $v_{\varepsilon}$ is positive and $v_{\varepsilon}(1)=\theta\left(1_{M}\right)+\varepsilon v\left(1_{B(H)}\right)$, the latter being invertible since $v_{\varepsilon}(1) \geq \theta\left(1_{M}\right)$ and $\delta<1$. Let $\eta=v_{\varepsilon}(1)$. Let $v^{\prime}(b)=\eta^{-1 / 2} v_{\varepsilon}(b) \eta^{-1 / 2}$, which is unital and positive. Equivalently we have $v_{\varepsilon}(b)=\eta^{1 / 2} v^{\prime}(b) \eta^{1 / 2}$, and hence by (5.1)

$$
\theta=\eta^{1 / 2} v^{\prime} u^{\prime} \eta^{1 / 2}-\varepsilon f(\cdot) v(1) .
$$

This implies (we write $\eta^{1 / 2} v^{\prime} u^{\prime} \eta^{1 / 2}-v^{\prime} u^{\prime}=\left(\eta^{1 / 2}-1\right) v^{\prime} u^{\prime} \eta^{1 / 2}+v^{\prime} u^{\prime}\left(\eta^{1 / 2}-1\right)$ )

$$
\left\|\theta-v^{\prime} u^{\prime}\right\| \leq\left\|\eta^{1 / 2}-1\right\|\left\|\eta^{1 / 2}\right\|+\left\|\eta^{1 / 2}-1\right\|+\varepsilon\|v(1)\|
$$

Since $1-\delta \leq \eta(1) \leq 1+\delta+\varepsilon\|v(1)\|$, we have $\left\|\eta^{1 / 2}-1\right\| \leq(\delta+\varepsilon\|v\|)^{1 / 2}$ and $\left\|\eta^{1 / 2}\right\| \leq(1+\delta+$ $\varepsilon\|v\|)^{1 / 2} \leq 2^{1 / 2}$. The announced bound follows.

Remark 5.3. Let $M$ be a von Neumann algebra. Assume that we have a net of pairs of maps $u_{\varepsilon}: M \rightarrow B(H), v_{\varepsilon}: B(H) \rightarrow M$ (here for convenience $\varepsilon$ is used for the index of the net) such that the composition $\theta_{\varepsilon}=v_{\varepsilon} u_{\varepsilon}$ satisfies $\left\|\theta_{\varepsilon}(1)-1\right\|_{M} \rightarrow 0$ and $\theta_{\varepsilon}(x) \rightarrow x$ weak* for any $x \in M$.

Note that this holds in particular if $v_{\varepsilon} u_{\varepsilon}=I d_{M}$ independently of $\varepsilon$.

Assume moreover that $u_{\varepsilon}, u_{\varepsilon}$ are both positive, that $\sup _{\varepsilon}\left\|v_{\varepsilon}\right\|<\infty$ and that $u_{\varepsilon}$ is normal. Then there is a factorization $I d_{M}=v u$ as in (1.1) with $u$ normal and $u, v$ both unital positive. Indeed, this follows from Lemma 5.2 and Proposition 5.1 .

In particular, having $I d_{M}=v^{\prime} u^{\prime}$ as in (1.1) with $u^{\prime}, v^{\prime}$ merely positive and $u^{\prime}$ normal implies the existence of $I d_{M}=v u$ as in (1.1) with $u$ normal and $u, v$ both unital positive.

Furthermore we will show at the end of the proof of Theorem 1.11 that the latter factorization implies that $I d_{M}$ admits the seemingly injective factorization of Definition 1.1.

As before, for any map $u: A \rightarrow M$ from a $C^{*}$-algebra to a von Neumann one we denote by $\ddot{u}: A^{* *} \rightarrow M$ the canonical normal extension of $u$ to $A^{* *}$, so that $\ddot{u} i_{A}=u$.

We next state an easy fact that will help us to relate our main topic to lifting problems.

Lemma 5.4. If a von Neumann algebra $M$ is $Q W E P$, there is a unital $C^{*}$-algebra $W$ and a surjective unital $*$-homomorphism $q: W \rightarrow M$ that admits for some $H$ a factorization of the form

$$
q: W \stackrel{w_{1}}{\longrightarrow} B(H) \stackrel{w_{2}}{\longrightarrow} M
$$

where $w_{1}$ is a unital $*$-homomorphism and $w_{2}$ is a unital c.p. map (and hence $\left\|w_{2}\right\|_{c b}=1$ ).

Proof. Let $q: W \rightarrow M$ be a surjective unital $*$-homomorphism. Let $i_{W}: W \rightarrow W^{* *}$ denote the inclusion. Let $\ddot{q}: W^{* *} \rightarrow M$ denote the normal $*$-homomorphism extending $q$ so that $\ddot{q} i_{W}=q$. If $W$ is WEP, the inclusion $i_{W}: W \rightarrow W^{* *}$ factors as $i_{W}: W \stackrel{w_{1}}{\longrightarrow} B(H) \stackrel{v_{2}}{\longrightarrow} W^{* *}$, with $w_{1}$ unital $*$-homomorphism and $v_{2}$ unital c.p. Let $w_{2}=\ddot{q} v_{2}: B(H) \rightarrow M$. We have then $q=w_{2} w_{1}$. 
The next lemma is a simple consequence of Corollary 2.6.

Lemma 5.5. Let $M$ be a von Neumann algebra that is QWEP. Let $T: M \rightarrow M$ be a finite rank normal map. For some $H$ there is a factorization of $T$ of the form

$$
T: M \stackrel{u}{\longrightarrow} B(H) \stackrel{v}{\longrightarrow} M
$$

where $u$ is normal, finite rank with $\|u\|=\|T\|$ and $v$ is a unital c.p. map (and hence $\|v\|=1$ ). If $T$ is in addition unital positive, then we can ensure that $u$ is also unital positive.

Proof. Let $W, q, w_{1}, w_{2}$ be as in the preceding lemma. Let $E=T(M) \subset M=q(W)$. By Corollary 2.6 there is a contractive unital positive lifting $r: E \rightarrow W$. Let $u=w_{1} r T$ and $v=w_{2}$. Then since $T$ is normal (resp. and unital positive) and $\operatorname{dim}(E)<\infty$ the map $u$ is normal (resp. and unital positive) with $\|u\| \leq\|T\|$, while $v$ is u.c.p. and $T=v u$. The latter implies $\|T\| \leq\|v\|\|u\| \leq\|u\|$.

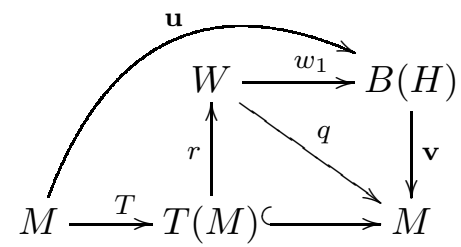

Before proving Theorem 1.11, we start by some preliminary remarks.

Remark 5.6. Consider the implication (i) $\Rightarrow$ (ii) in Theorem 1.11, By structural results we may assume that $M$ is $\sigma$-finite. Indeed, by [57, Chap. 5, prop. 1.40] a general $M$ is isomorphic to a direct sum of a family of algebras of the form $M=B(H) \bar{\otimes} N$ with $N \sigma$-finite. For such an $M$, there is a net of projections $\left(p_{\alpha}\right)$ in $M$ tending weak* to 1 such that $p_{\alpha} M p_{\alpha}$ is $\sigma$-finite, or equivalently the unital subalgebra $M_{\alpha}=p_{\alpha} M p_{\alpha} \oplus \mathbb{C}\left(1-p_{\alpha}\right)$ is $\sigma$-finite and is the range of a unital normal c.p. projection $Q_{\alpha}$ defined by $Q_{\alpha}(x)=p_{\alpha} x p_{\alpha}+f(x)\left(1-p_{\alpha}\right)$ where $f$ is a normal state on $M$. Then $Q_{\alpha}$ tends pointwise weak* to $I d_{M}$ and the range of each $Q_{\alpha}$ is $\sigma$-finite. Moreover, if $M$ is seemingly injective, so is $Q_{\alpha}(M)$ by Remark 1.6. Thus to show that $M$ has the weak* PAP it clearly suffices to show that each $Q_{\alpha}(M)$ has it, and the latter are $\sigma$-finite.

Remark 5.7. Consider again the implication (i) $\Rightarrow$ (ii) in Theorem 1.11. Assume $M \sigma$-finite. A tempting approach to prove this without using standard forms is to first prove this assuming $M$ finite, from which the semi-finite case follows easily (using $p M p$ for finite projections $p$ ), and then to invoke Takesaki's duality theorem to pass from the semi-finite case to the general one. This roughly produces a general $\sigma$-finite algebra as the range of a unital c.p. projection on a semi-finite one. However since the latter projection is in general not normal, this route does not seem to go through to prove the weak* PAP. (We fell into that trap in a previous version of this paper.) In [62], to show that injectivity implies the weak* CPAP, Wassermann uses this path to prove that injectivity implies (iii) in Theorem 6.1 and then he relies on (iii) $\Rightarrow$ (i) in Theorem 6.1 (previously proved by Effros-Lance [23]). In the seemingly injective context, the analogue of the latter implication is what we are after, so this approach no longer seems to work. This explains our recourse to standard forms. Using the latter, we can give an argument covering the case of a general $\sigma$-finite algebra. Nevertheless, we include the finite case in the proof below because we feel it will be much easier for the reader to follow the general case after this warm up.

Proof of Theorem 1.11. Assume (i). Then $M$ is isometric to a (Banach) quotient of some $B(H)$ and hence is QWEP by Kirchberg's results (see e.g. [45] or [52, Th. 15.5]). We will derive the matricial weak* PAP by the same kind of argument as the one which shows that injective von 
Neumann algebras have the weak* CPAP (see Remark 1.16 for references).

By Remark [5.6] we may assume that $M$ is $\sigma$-finite.

We will first prove (i) $\Rightarrow$ (ii) in the finite case. Then in the second part of the proof we will show how the argument can be adapted to cover the general case using the standard form of $M$.

Thus we first consider a tracial probability space $(M, \tau)$. Let $B(H), u, v$ be as in (1.1) with $u, v$ both unital and positive (or equivalently unital and contractive). Let $\mathcal{H}=H \oplus H \oplus \cdots$. We may embed $B(H)$ in $B(\mathcal{H})$ acting diagonally with infinite multiplicity, and we have a unital c.p. projection from $B(\mathcal{H})$ to this copy of $B(H)$. By Proposition 4.1 (with $B=B(H)$ ) and Remark 4.4 we may assume that we have an ultrafilter $\mathcal{U}$ refining a net of finite rank operators $h_{\alpha} \in C_{+}$such that for all $b \in B(H)$

$$
\tau\left(v(b)^{*} v(b)\right) \leq \lim _{\mathcal{U}} \operatorname{tr}\left(b^{*} h_{\alpha} b h_{\alpha}\right) .
$$

Here since we assume (i) $v$ is unital and c.p. and hence satisfies (4.6) by Remark 4.2 and (4.4). However, we wish to record for future use (at the end of the proof) that this also holds for all positive $v: B(H) \rightarrow M$ by Lemma 4.3 and (4.4).

Let $b^{\prime}=u(U)$ with $U$ unitary in $M$. We have $v\left(b^{\prime}\right)=U$ and hence $1=\tau\left(v\left(b^{\prime}\right)^{*} v\left(b^{\prime}\right)\right) \leq$ $\lim _{\mathcal{U}} \operatorname{tr}\left(b^{\prime *} h_{\alpha} b^{\prime} h_{\alpha}\right) \leq 1$ so that $\tau\left(v\left(b^{\prime}\right)^{*} v\left(b^{\prime}\right)\right)=\lim \operatorname{tr}\left(b^{\prime *} h_{\alpha} b^{\prime} h_{\alpha}\right)$.

(Therefore $\lim \left\|b^{\prime} h_{\alpha}-h_{\alpha} b^{\prime}\right\|_{2}=0$, but actually we will not use this.)

Let $\langle\langle b, b\rangle\rangle=\lim _{\mathcal{U}} \operatorname{tr}\left(b^{*} h_{\alpha} b h_{\alpha}\right)-\tau\left(v(b)^{*} v(b)\right)$ for $b \in B(H)$. By Cauchy-Schwarz for the inner product $\langle\langle b, b\rangle\rangle$ we have

$$
\left|\left\langle\left\langle b^{\prime}, b\right\rangle\right\rangle\right|^{2} \leq\left\langle\left\langle b^{\prime}, b^{\prime}\right\rangle\right\rangle\langle\langle b, b\rangle\rangle
$$

and hence $\left\langle\left\langle b, b^{\prime}\right\rangle\right\rangle=0$ for any $b \in B(H)$. Since $M$ is spanned by its unitaries this remains true for any $b^{\prime} \in u(M)$. In particular

$$
\tau\left(v(b)^{*} v(b)\right)=\lim _{\mathcal{U}} \operatorname{tr}\left(b^{*} h_{\alpha} b h_{\alpha}\right)
$$

for any $b \in u(M)$. Note that by polarization (5.3) implies $\tau\left(v(b)^{*} v(a)\right)=\lim \operatorname{tr}\left(b^{*} h_{\alpha} a h_{\alpha}\right)$ for any pair $a, b \in u(M)$. Moreover, for any $b \in B$ and $x \in M$, let $b^{\prime}=u(x)$. Since $\left\langle\left\langle b, b^{\prime}\right\rangle\right\rangle=0$ we have

$$
\tau\left(x^{*} v(b)\right)=\lim _{\mathcal{U}} \operatorname{tr}\left(u(x)^{*} h_{\alpha} b h_{\alpha}\right) .
$$

Recall that $u: M \rightarrow B(H)$ is a normal unital positive isometric map and all the $h_{\alpha}$ 's are of finite rank. Since $u$ is normal and $h_{\alpha} b h_{\alpha} \in B(H)_{*}$, for any given $b \in M$, the linear form $x \mapsto$ $\overline{\operatorname{tr}\left(u(x)^{*} h_{\alpha} b h_{\alpha}\right)}$ is in $M_{*}$. We identify $M_{*}$ with $L_{1}(\tau)$. Note that since $u$ is positive $u(x)^{*}=u\left(x^{*}\right)$. Thus we have a (uniquely defined) linear map $V_{\alpha}: B(H) \rightarrow M_{*}$ such that

$$
\forall x \in M \forall b \in B(H) \quad \operatorname{tr}\left(u(x)^{*} h_{\alpha} b h_{\alpha}\right)=\tau\left(x^{*} V_{\alpha}(b)\right) .
$$

Since $h_{\alpha}$ has finite rank, so has $V_{\alpha}$. Since $u$ is positive, $\tau\left(x^{*} V_{\alpha}(b)\right) \geq 0$ for any $x \in M_{+}, b \in B(H)_{+}$, and hence $V_{\alpha}$ is positive. By (5.4)

$$
\tau\left(x^{*} v(b)\right)=\lim \tau\left(x^{*} V_{\alpha}(b)\right)
$$

for any $x \in M, b \in B(H)$ and hence denoting by $j: M \rightarrow M_{*}$ the canonical embedding we have $V_{\alpha}(b)-j(v(b)) \rightarrow 0$ with respect to $\sigma\left(M_{*}, M\right)$ or equivalently weakly in $M_{*}$. Passing to convex combinations of the $V_{\alpha}$ 's we may assume that $\left\|V_{\alpha}\left(1_{B(H)}\right)-j\left(1_{M}\right)\right\|_{M_{*}} \rightarrow 0$ (and also that $\left\|V_{\alpha}(b)-j(v(b))\right\|_{M_{*}} \rightarrow 0$ for any $\left.b \in B(H)\right)$. Assume for a moment that $V_{\alpha}(1)^{-1}$ exists and lies in $M$. Then if we set

$$
T_{\alpha}(b)=V_{\alpha}(1)^{-1 / 2} V_{\alpha}(b) V_{\alpha}(1)^{-1 / 2},
$$


the map $T_{\alpha}: B(H) \rightarrow M$ is positive, unital (and hence contractive), of finite rank and we have

$$
\tau\left(x^{*} v(b)\right)=\lim \tau\left(x^{*} V_{\alpha}(1)^{1 / 2} T_{\alpha}(b) V_{\alpha}(1)^{1 / 2}\right) .
$$

Since $V_{\alpha}(1) \rightarrow 1$ in $L_{1}(\tau)$ we have $\lim \left\|V_{\alpha}(1)^{1 / 2}-1\right\|_{L_{2}(\tau)} \rightarrow 0$; indeed this follows from the PowersStørmer inequality (see e.g. [19, Prop. 1.2.1], but in the present case since $V_{\alpha}(1)^{1 / 2}$ and 1 commute this could be reduced to the commutative case). In any case (for $x \in M, b \in B(H)$ )

$$
\tau\left(x^{*} v(b)\right)=\lim \tau\left(x^{*} T_{\alpha}(b)\right) .
$$

This shows that $T_{\alpha}(b) \rightarrow v(b)$ for $\sigma(M, M)$ and hence since $M$ is dense in $M_{*}$ and $\left(T_{\alpha}\right)$ is equicontinuous, $T_{\alpha}(b) \rightarrow v(b)$ for $\sigma\left(M, M_{*}\right)$.

Now let $H_{\alpha} \subset H$ be the (finite dimensional) range of $h_{\alpha}$ and let $p_{\alpha} \in B\left(H, H_{\alpha}\right)$ denote the orthogonal projection onto $H_{\alpha}$. We define $u_{\alpha}: M \rightarrow B\left(H_{\alpha}\right)$ by setting $u_{\alpha}(x)=p_{\alpha} u(x) p_{\alpha}^{*}$, and $v_{\alpha}(y)=T_{\alpha}\left(p_{\alpha}^{*} y p_{\alpha}\right)$. Note that since $p_{\alpha}^{*} p_{\alpha} h_{\alpha}=h_{\alpha} p_{\alpha}^{*} p_{\alpha}=h_{\alpha}$, by (5.5) and (5.6) we have $T_{\alpha}\left(p_{\alpha}^{*} p_{\alpha} b p_{\alpha}^{*} p_{\alpha}\right)=T_{\alpha}(b)$ for any $b \in B(H)$, and hence $v_{\alpha}\left(u_{\alpha}(x)\right)=T_{\alpha}(u(x))$ for any $x \in M$. This shows that $v_{\alpha}\left(u_{\alpha}(x)\right)$ tends weak* to $v(u(x))=x$. Let $n(\alpha)=\operatorname{dim}\left(H_{\alpha}\right)$. We may identify $B\left(H_{\alpha}\right)$ with $M_{n(\alpha)}$. Then $u_{\alpha}$ is normal, $u_{\alpha}, v_{\alpha}$ are both unital and positive (and hence contractive). Thus $M$ satisfies (ii). The only drawback is that we assumed $V_{\alpha}(1)^{-1} \in M$. This can be fixed easily: we pick a state $f$ on $B(H)$, we replace $V_{\alpha}$ by $V_{\alpha, \varepsilon}$ defined by $V_{\alpha, \varepsilon}(b)=V_{\alpha}(b)+\varepsilon f(b) 1_{M}(b \in B(H))$ so that $V_{\alpha, \varepsilon}(1)^{-1} \leq \varepsilon^{-1} 1_{M}$, and we let $\varepsilon>0$ tend to zero as part of our net. This completes the proof that (i) $\Rightarrow$ (ii) in the finite case.

We will now prove the same implication (i) $\Rightarrow$ (ii) for a general $M$. Assume (i). Again, by Remark 5.6 we may assume $M \sigma$-finite so that $M$ admits a faithful normal state $\varphi$.

We will use Corollary 3.7. Let $\xi=\xi_{\varphi}$. Note $\|\xi\|=1$. Let $s(x, x)=\langle\xi, J x J x \xi\rangle(x \in M)$. Let $B=B(H)$. We claim that for any self-adjoint tensor $t=\sum \overline{b_{j}} \otimes b_{j}$ in $\bar{B} \otimes B$, we have

$$
\sum\left\langle\xi, J v\left(b_{j}\right) J v\left(b_{j}\right) \xi\right\rangle \leq\left\|\sum \overline{b_{j}} \otimes b_{j}\right\|_{\min } .
$$

Indeed, by Remark 3.3 and (4.4) we have

$$
\sum\left\langle\xi, J v\left(b_{j}\right) J v\left(b_{j}\right) \xi\right\rangle \leq\left\|\sum \overline{v\left(b_{j}\right)} \otimes v\left(b_{j}\right)\right\|_{\max } \leq\left\|\sum \overline{b_{j}} \otimes b_{j}\right\|_{\max }=\left\|\sum \overline{b_{j}} \otimes b_{j}\right\|_{\min } .
$$

By the argument used in Proposition 4.1, our claim implies that there is a net $\left(h_{\alpha}\right)$ in $C_{+}$such that

$$
\forall b \in B \quad\langle\xi, J v(b) J v(b) \xi\rangle \leq \lim _{\mathcal{U}} \operatorname{tr}\left(b^{*} h_{\alpha} b h_{\alpha}\right),
$$

where $\mathcal{U}$ is an ultrafilter refining our net. Indeed, we may use the diagonal embedding $B(H) \subset$ $B(H \oplus H \oplus \cdots)$ to replace $H$ by $H \oplus H \oplus \cdots$ in order to replace the finitely supported probabilities on $\mathcal{P} \times C_{+}$by singly supported ones. (Actually this is not essential, but it makes the argument slightly easier to follow.)

Since $v u=I d_{M}$, we have

$$
\forall x \in M \quad\langle\xi, J x J x \xi\rangle \leq \lim _{\mathcal{U}} \operatorname{tr}\left(u(x)^{*} h_{\alpha} u(x) h_{\alpha}\right),
$$

We will now show that equality holds in (5.8). Let $s^{\prime}$ be the sesquilinear form defined on $M$ by

$$
s^{\prime}(y, x)=\lim _{\mathcal{U}} \operatorname{tr}\left(u(y)^{*} h_{\alpha} u(x) h_{\alpha}\right) .
$$

Note that $s^{\prime}$ is bipositive and $s^{\prime}(1,1)=1$. Since $s$ is self-polar and $s \leq s^{\prime}$ on the diagonal, we must have $s=s^{\prime}$ by Theorem 3.8. Thus we have

$$
\forall y, x \in M \quad\langle\xi, J y J x \xi\rangle=\lim _{\mathcal{U}} \operatorname{tr}\left(u(y)^{*} h_{\alpha} u(x) h_{\alpha}\right) .
$$


Since $h_{\alpha}$ has finite rank, there is a finite rank projection $P_{\alpha}$ such that $P_{\alpha} h_{\alpha}=h_{\alpha} P_{\alpha}=h_{\alpha}$, and hence $\operatorname{tr}\left(u(y)^{*} h_{\alpha} u(x) h_{\alpha}\right)=\operatorname{tr}\left(P_{\alpha} u(y)^{*} P_{\alpha} h_{\alpha} P_{\alpha} u(x) P_{\alpha} h_{\alpha}\right)$. Therefore there is a positive unital finite rank map $T_{\alpha}: \bar{M} \rightarrow M_{*}$ such that

$$
\forall y, x \in M \quad T_{\alpha}(\bar{y})(x)=\operatorname{tr}\left(u(y)^{*} h_{\alpha} u(x) h_{\alpha}\right) .
$$

We have $\lim _{\mathcal{U}} T_{\alpha}(\bar{y})(x)=\langle\xi, J y J x \xi\rangle$. In particular $T_{\alpha}(\overline{1}) \rightarrow \varphi$ with respect to the weak topology $\sigma\left(M_{*}, M\right)$. Passing to convex linear combinations of the $T_{\alpha}$ 's we may assume that $\left\|T_{\alpha}(\overline{1})-\varphi\right\|_{M_{*}}$ tends to 0 .

Let $\varepsilon>0$. Consider the positive map $T_{\alpha, \varepsilon}: \bar{M} \rightarrow M_{*}$ defined by

$$
T_{\alpha, \varepsilon}(\bar{y})=T_{\alpha}(\bar{y})+\varepsilon \varphi\left(y^{*}\right) \varphi .
$$

The advantage of this perturbation of $T_{\alpha}$ is that $T_{\alpha, \varepsilon}(\overline{1})$ is faithful (since it dominates $\varepsilon \varphi$ ). Let $\xi_{\alpha, \varepsilon}$ be the (unique) vector in $P^{\natural}$ such that $T_{\alpha, \varepsilon}(\overline{1})(x)=\left\langle\xi_{\alpha, \varepsilon}, x \xi_{\alpha, \varepsilon}\right\rangle$. We now make $\varepsilon$ tending to 0 as part of our new net $\left(T_{\alpha, \varepsilon}\right)$ indexed by $\alpha, \varepsilon$. Clearly $\lim \left\|T_{\alpha, \varepsilon}(\overline{1})-\varphi\right\|_{M_{*}}=0$. By the generalized Powers-Størmer inequality (see [27, Lemma 2.10]) we have $\left\|\xi_{\alpha, \varepsilon}-\xi\right\| \rightarrow 0$.

We now apply Corollary 3.7 to the faithful form $T_{\alpha, \varepsilon}(\overline{1})$. Since $T_{\alpha, \varepsilon}$ is positive, there is a uniquely defined positive unital finite rank map $V_{\alpha, \varepsilon}: M \rightarrow M$ such that $T_{\alpha, \varepsilon}(\bar{y})(x)=\left\langle\xi_{\alpha, \varepsilon}, J V_{\alpha, \varepsilon}(y) J x \xi_{\alpha, \varepsilon}\right\rangle$. Since $\left\|\xi_{\alpha, \varepsilon}-\xi\right\| \rightarrow 0$, we have

$$
\lim \left|\left\langle\xi_{\alpha, \varepsilon}, J V_{\alpha, \varepsilon}(y) J x \xi_{\alpha, \varepsilon}\right\rangle-\left\langle\xi, J V_{\alpha, \varepsilon}(y) J x \xi\right\rangle\right|=0 .
$$

Thus since $\lim T_{\alpha, \varepsilon}(\bar{y})(x)=\lim T_{\alpha}(\bar{y})(x)$ we obtain

$$
\lim \left\langle\xi, J V_{\alpha, \varepsilon}(y) J x \xi\right\rangle=\langle\xi, J y J x \xi\rangle,
$$

and hence $\left(\right.$ since $\left\langle x_{1} \xi, J y J x_{2} \xi\right\rangle=\left\langle\xi, J y J x_{1}^{*} x_{2} \xi\right\rangle$ )

$$
\forall x_{1}, x_{2} \in M \forall y \in M \quad \lim \left\langle x_{1} \xi, J V_{\alpha, \varepsilon}(y) J x_{2} \xi\right\rangle=\left\langle x_{1} \xi, J y J x_{2} \xi\right\rangle .
$$

Since $V_{\alpha, \varepsilon}$ is unital and positive it is contractive. By (5.10) the form $(y, x) \mapsto T_{\alpha, \varepsilon}(\bar{y})(x)$ is separately normal (since $u$ and $\varphi$ are normal). Let $L=\left\{\varphi_{x} \mid x \in M\right\} \subset M_{*}$ with $\varphi_{x}(\bar{y})=\langle\xi, J y J x \xi\rangle$. The linear span of the set $L$ is norm dense in $\overline{M_{*}}$ (because by Remark $3.2 \overline{M \xi}=H$ which implies $\left.L^{\perp}=\{0\}\right)$. Therefore $V_{\alpha, \varepsilon}$ is normal and (5.11) implies that $\lim V_{\alpha, \varepsilon}(y)=y$ for the weak topology $\sigma\left(M, M_{*}\right)$. This proves that (i) $\Rightarrow$ (iii) (since we already noted that QWEP follows from (i)). We recorded this argument for later use in Theorem 5.9 , but we actually want to prove (i) $\Rightarrow$ (ii). This requires a little more work. Assume (i) still.

We start again from

$$
\forall x \in M \quad\langle\xi, J x J x \xi\rangle \leq \lim _{\mathcal{U}} \operatorname{tr}\left(u(x)^{*} h_{\alpha} u(x) h_{\alpha}\right),
$$

Arguing exactly as for (5.4) we deduce from (5.7) and (5.9) that

$$
\forall x \in M, \forall b \in B \quad\langle\xi, J v(b) J x \xi\rangle=\lim _{\mathcal{U}} \operatorname{tr}\left(b^{*} h_{\alpha} u(x) h_{\alpha}\right) .
$$

Since $u$ is normal and $h_{\alpha} b^{*} h_{\alpha} \in B(H)_{*}$ for any $b \in B$, the linear form

$$
\theta_{\alpha}(\bar{b}): x \mapsto \operatorname{tr}\left(b^{*} h_{\alpha} u(x) h_{\alpha}\right)
$$

is in $M_{*}$. Moreover $\theta_{\alpha}: \bar{B} \rightarrow M_{*}$ is a (linear) positive map of finite rank. Recall $P_{\alpha}$ are finite rank projections in $B(H)$ such that $h_{\alpha}=P_{\alpha} h_{\alpha} P_{\alpha}$. Note that

$$
\theta_{\alpha}(\bar{b})=\theta_{\alpha}\left(\overline{P_{\alpha} b P_{\alpha}}\right) .
$$


Let $\varepsilon>0$. Let $f_{\alpha}$ be a normal state on $B$ such that $f_{\alpha}\left(P_{\alpha}\right)=1$. Let

$$
\theta_{\alpha, \varepsilon}(\bar{b})=\theta_{\alpha}(\bar{b})+\varepsilon f_{\alpha}\left(b^{*}\right) \varphi .
$$

We still have

$$
\theta_{\alpha, \varepsilon}(\bar{b})=\theta_{\alpha, \varepsilon}\left(\overline{P_{\alpha} b P_{\alpha}}\right)
$$

and $\theta_{\alpha, \varepsilon}: \bar{B} \rightarrow M_{*}$ is positive with finite rank, but in addition $\theta_{\alpha, \varepsilon}(\overline{1})$ is faithful.

Let $\xi_{\alpha, \varepsilon}$ be the (unique) vector in $P^{\natural}$ such that $\theta_{\alpha, \varepsilon}(\overline{1})(x)=\left\langle\xi_{\alpha, \varepsilon}, x \xi_{\alpha, \varepsilon}\right\rangle$. We now apply Corollary 3.7 to the faithful form $\theta_{\alpha, \varepsilon}(\overline{1})$. Since $\theta_{\alpha, \varepsilon}$ is positive, there is a uniquely defined positive unital linear map $W_{\alpha, \varepsilon}: B \rightarrow M$ such that

$$
\theta_{\alpha, \varepsilon}(\bar{b})(x)=\left\langle\xi_{\alpha, \varepsilon}, J W_{\alpha, \varepsilon}(b) J x \xi_{\alpha, \varepsilon}\right\rangle .
$$

We will make $\varepsilon$ tending to 0 as part of our new nets indexed by $\alpha, \varepsilon$. Since $\theta_{\alpha, \varepsilon}(\overline{1}) \rightarrow \varphi$ weakly in $M_{*}$, we may pass to convex combinations to ensure that $\theta_{\alpha, \varepsilon}(\overline{1}) \rightarrow \varphi$ in norm in $M_{*}$. Then as before $\left\|\xi_{\alpha, \varepsilon}-\xi\right\| \rightarrow 0$.

Let $T_{\alpha, \varepsilon}=W_{\alpha, \varepsilon} u$. By (5.14) we have $W_{\alpha, \varepsilon}(b)=W_{\alpha, \varepsilon}\left(P_{\alpha} b P_{\alpha}\right)$ for all $b \in B$. In particular, $W_{\alpha, \varepsilon}(1)=W_{\alpha, \varepsilon}\left(P_{\alpha}\right)=1$. Let $H_{\alpha}=P_{\alpha}(H)$. Let $i_{\alpha}: H_{\alpha} \rightarrow H$ denote the inclusion map. Of course $i_{\alpha}^{*}: H \rightarrow H_{\alpha}$ is essentially the same as $P_{\alpha}$ : more precisely $i_{\alpha} i_{\alpha}^{*}=P_{\alpha}$. We define $u_{\alpha}: M \rightarrow B\left(H_{\alpha}\right)$ by $u_{\alpha}(x)=i_{\alpha}^{*} u(x) i_{\alpha}(x \in M)$, and $v_{\alpha, \varepsilon}: B\left(H_{\alpha}\right) \rightarrow M$ by $v_{\alpha, \varepsilon}(b)=W_{\alpha, \varepsilon}\left(i_{\alpha} b i_{\alpha}^{*}\right)$ for any $b \in B\left(H_{\alpha}\right)$. Clearly

$$
T_{\alpha, \varepsilon}=W_{\alpha, \varepsilon} u=v_{\alpha, \varepsilon} u_{\alpha}
$$

Therefore the maps in the net $\left(T_{\alpha, \varepsilon}\right)$ have the factorization required in (ii) in Theorem 1.11, Thus to conclude it suffices to verify that for any $y \in M$

$$
\text { weak }^{*} \lim T_{\alpha, \varepsilon}(y)=\text { weak }^{*} \lim W_{\alpha, \varepsilon} u(y)=y
$$

or equivalently that

$$
\text { weak }^{*} \lim J W_{\alpha, \varepsilon} u(y) J=J y J .
$$

Since the set $L$ is total in $\overline{M_{*}}$, it suffices to show that $\lim \varphi_{x}\left(\overline{W_{\alpha, \varepsilon} u(y)}\right)=\varphi_{x}(\bar{y})$ for any $x, y \in M$. We have (since $\left\|\xi_{\alpha, \varepsilon}-\xi\right\| \rightarrow 0$ ) by (5.15) and (5.13)

$$
\begin{aligned}
\lim \varphi_{x}\left(\overline{W_{\alpha, \varepsilon} u(y)}\right) & =\lim \left\langle\xi_{\alpha, \varepsilon}, J W_{\alpha, \varepsilon} u(y) J x \xi_{\alpha, \varepsilon}\right\rangle=\lim \theta_{\alpha, \varepsilon}(\overline{u(y)})(x)=\lim \theta_{\alpha}(\overline{u(y)})(x) \\
& =\lim \operatorname{tr}\left(u(y)^{*} h_{\alpha} u(x) h_{\alpha}\right)=\langle\xi, J y J x \xi\rangle=\varphi_{x}(\bar{y}) .
\end{aligned}
$$

This completes the proof of (i) $\Rightarrow$ (ii).

Assume (ii) then the weak* PAP is clear. In addition (ii) implies that $I d_{M}$ factors as

$$
I d_{M}=v u: M \stackrel{u}{\longrightarrow} \ell_{\infty}\left(\left\{M_{n(\alpha)}\right\}\right) \stackrel{v}{\longrightarrow} M
$$

where $v, u$ are unital positive contractions (and $u$ is normal). Indeed, when $x \in M$ and $\left(a_{\alpha}\right) \in$ $\ell_{\infty}\left(\left\{M_{n(\alpha)}\right\}\right)$, we may take $u(x)=\left(u_{\alpha}(x)\right)$ and $v\left(\left(a_{\alpha}\right)\right)=$ weak $^{*} \lim _{\mathcal{U}} v_{\alpha}\left(a_{\alpha}\right)$ for some suitable ultrafilter $\mathcal{U}$. It follows that $M$ is isometric to a quotient Banach space of some $B(H)$ and hence by a result of Kirchberg (see [52, Th. 15.5]) $M$ is QWEP. Thus (ii) $\Rightarrow$ (iii).

Assume (iii). Let $\left(T_{\alpha}\right)_{\alpha \in I}$ be a net of normal finite rank unital maps tending pointwise weak* to $I d_{M}$. Let $T_{\alpha}=v_{\alpha} u_{\alpha}$ be a factorization through $B\left(H_{\alpha}\right)$ as in Lemma 5.5. Let $x \in M$ and $\left(a_{\alpha}\right) \in \ell_{\infty}\left(\left\{B\left(H_{\alpha}\right)\right\}\right)$. We define $u: M \rightarrow \ell_{\infty}\left(\left\{B\left(H_{\alpha}\right)\right\}\right)$ by $u(x)=\left(u_{\alpha}(x)\right)_{\alpha \in I}$ and $v\left(\left(a_{\alpha}\right)\right)=$ 
weak ${ }^{*} \lim _{\mathcal{U}} v_{\alpha}\left(a_{\alpha}\right)$ for some suitable ultrafilter $\mathcal{U}$. This gives us a factorization of $I d_{M}$ of the form $M \stackrel{u}{\longrightarrow} \ell_{\infty}\left(\left\{B\left(H_{\alpha}\right)\right\}\right) \stackrel{v}{\longrightarrow} M$, with $u, v$ as required. Clearly $u^{*}\left(\ell_{1}\left(\left\{B\left(H_{\alpha}\right)_{*}\right\}\right) \subset M_{*}\right.$, and hence $u$ is normal. Since we may obviously replace $\ell_{\infty}\left(\left\{B\left(H_{\alpha}\right)\right\}\right)$ by $B(H)$ with $H=\oplus H_{\alpha}$, this shows that $M$ is seemingly injective.

For the last sentence in Theorem 1.11, suppose:

(i) ' We have $I d_{M}=v u$ assuming merely that $u$ and $v$ are positive maps, with $u$ normal.

Then, by Remark [5.3, $I d_{M}$ has a factorization as in (1.1) with unital positive $u, v$ and $u$ normal. A fortiori $\|u\|=\|v\|=1$. As we already observed, the mere positivity of $v$ is enough to guarantee (5.2). But the latter was the only assumption needed on $v$ to complete the preceding argument for (i) $\Rightarrow$ (ii). This shows (i) ' $\Rightarrow$ (ii) and hence (i)' $\Rightarrow$ (i), and since the converse is trivial the proof is complete.

Remark 5.8. The preceding proof of (iii) $\Rightarrow$ (i) (resp. with $I$ countable) shows that any QWEP $M$ with the weak* BAP is isomorphic to a complemented subspace of $B(H)$ (resp. $B\left(\ell_{2}\right)$ ). More precisely, in the weak* BAP case, Lemma 5.5 gives us a factorization as in (1.1) but in which the map $u$ is merely bounded and normal.

We end this section with a characterization of the weak* PAP. It is not clear that it is really different from that of Theorem 1.11, because we do not know whether the weak* PAP implies QWEP, as is the case in the c.p. case for injectivity.

Theorem 5.9. Consider a $\sigma$-finite von Neumann algebra $M \subset B(H)$ in standard form. Let $\varphi$ be a faithful normal state on $M$ and let $s$ be the associated self-polar form on $M$. The following are equivalent:

(i) $M$ has the weak* PAP.

(ii) There is a net of bipositive, separately normal finite rank forms $s_{\alpha}$ tending pointwise to $s$.

Proof. Assume (i). Let $\left(T_{\alpha}\right)$ be a net of unital positive finite rank normal maps tending to $I d_{M}$ pointwise weak* ${ }^{*}$ Let $s_{\alpha}(y, x)=s\left(T_{\alpha}(y), T_{\alpha}(x)\right)$. Since $s$ is separately weak* continuous, we have $s(x, x) \leq \liminf s_{\alpha}(x, x)$. (Indeed, we have by Cauchy-Schwarz $|s(x, x)|^{2}=\lim \left|s\left(x, T_{\alpha}(x)\right)\right|^{2} \leq$ $\lim \inf |s(x, x)|\left|s\left(T_{\alpha}(x), T_{\alpha}(x)\right)\right|$.) Thus if $\mathcal{U}$ is an ultrafilter refining the net we have $s(x, x) \leq s^{\prime}(x, x)$ with $s^{\prime}(x, x)=\lim _{\mathcal{U}} s_{\alpha}(x, x)$. Since $s^{\prime}$ is a bipositive normalized sesquilinear form we have $s^{\prime}=s$ by Theorem 3.8 whence (ii).

Assume (ii). We will repeat the proof of the implication (i) $\Rightarrow$ (iii) in Theorem 1.11, so we just sketch the argument. We replace the net $\left(s_{\alpha}\right)$ by $s_{\alpha, \varepsilon}(y, x)=(1+\varepsilon)^{-1}\left(s_{\alpha}(y, x)+\varepsilon \overline{\varphi(y)} \varphi(x)\right)$. Let $\varphi_{\alpha, \varepsilon}(x)=s_{\alpha, \varepsilon}(1, x)$. We define the unit vector $\xi_{\alpha, \varepsilon} \in P^{\natural}$ by $\varphi_{\alpha, \varepsilon}(x)=\left\langle\xi_{\alpha, \varepsilon}, x \xi_{\alpha, \varepsilon}\right\rangle$. Then, using Corollary 3.7, we define the unital positive (and hence contractive) finite rank map $V_{\alpha, \varepsilon}: M \rightarrow M$ by

$$
\forall x, y \in M \quad s_{\alpha, \varepsilon}(y, x)=\left\langle\xi_{\alpha, \varepsilon}, J V_{\alpha, \varepsilon}(y) J x \xi_{\alpha}\right\rangle .
$$

We have $\varphi_{\alpha, \varepsilon} \rightarrow \varphi$ weakly in $M_{*}$. Passing to convex combinations, we may assume that $\varphi_{\alpha, \varepsilon} \rightarrow \varphi$ in norm in $M_{*}$. By [27, Lemma 2.10] we have $\xi_{\alpha, \varepsilon} \rightarrow \xi$ in norm in $H$. This allows us to pass from $\lim \left\langle\xi_{\alpha, \varepsilon}, J V_{\alpha, \varepsilon}(y) J x \xi_{\alpha, \varepsilon}\right\rangle=s(y, x)$ to

$$
\lim \left\langle\xi, J V_{\alpha, \varepsilon}(y) J x \xi\right\rangle=s(y, x)=\langle\xi, J y J x \xi\rangle .
$$

By (5.11) again we conclude that $V_{\alpha, \varepsilon}(y) \rightarrow y$ for the weak* topology of $M$, which yields (i).

Remark 5.10. In [20], Connes and Jones studied an analogue of property (T) for a von Neumann algebra $M$. When $M$ is equipped with a standard finite trace $\tau$ the latter property (T) is an 
obstruction to the existence of certain approximations of the identity on $L_{2}(\tau)$ formed of compact c.p. maps. More generally, in Theorem 5.9 if $M$ has (T) then (ii) cannot hold if each bipositive form $s_{\alpha}$ defines a compact c.p. map from $M$ to $\overline{M_{*}}$. We suspect that some examples of $M$ with property $(\mathrm{T})$ can be found that enjoy a stronger property ruling out the same for compact positive maps; such examples would then fail the weak* PAP.

For completeness, we record here the following simple fact.

Lemma 5.11. Let $M$ be a von Neumann algebra. Assume that there is a uniformly bounded net of positive finite rank maps $\left(T_{\alpha}\right)$ that tend pointwise weak* to $I d_{M}$. Then $M$ has the weak* PAP.

Proof. We may assume $M \sigma$-finite and in standard form with a cyclic and separating unit vector $\xi \in P^{\natural}$. For any $\varepsilon>0$ we may replace $T_{\alpha}$ by $x \mapsto T_{\alpha}(x)+\varepsilon f(x) 1$ where $f$ is any fixed normal state, so that we may as well assume that $T_{\alpha}(1)$ is invertible for all $\alpha$. As earlier let $\xi_{\alpha} \in P^{\natural}$ such that $\left\langle\xi, J T_{\alpha}(1) J x \xi\right\rangle=\left\langle\xi_{\alpha}, x \xi_{\alpha}\right\rangle$. By Corollary 3.7 there is a unital positive finite rank normal map $T_{\alpha}^{\prime}: M \rightarrow M$ such that $\left\langle\xi, J T_{\alpha}(y) J x \xi\right\rangle=\left\langle\xi_{\alpha}, J T_{\alpha}^{\prime}(y) J x \xi_{\alpha}\right\rangle$ for any $y \in M$. Since $T_{\alpha}(1) \rightarrow 1$ weak $^{*}$, the normal forms $x \mapsto\left\langle\xi_{\alpha}, x \xi_{\alpha}\right\rangle$ tend pointwise to $x \mapsto\langle\xi, x \xi\rangle$ and are bounded in $M_{*}$. Passing to convex combinations, we may assume the convergence is in the norm of $M_{*}$. By [27, Lemma 2.10] we have $\xi_{\alpha} \rightarrow \xi$ in norm in $H$. It follows that

$$
\lim \left\langle\xi, J T_{\alpha}^{\prime}(y) J x \xi\right\rangle=\lim \left\langle\xi_{\alpha}, J T_{\alpha}^{\prime}(y) J x \xi_{\alpha}\right\rangle=\lim \left\langle\xi, J T_{\alpha}(y) J x \xi\right\rangle=\langle\xi, J y J x \xi\rangle .
$$

As before this implies that $T_{\alpha}^{\prime}(y) \rightarrow y$ weak*.

\section{Comparison with injectivity}

Our goal here is to highlight the analogy of our results to the previous ones on injectivity and semidiscreteness, that came out of [19]. Connes's ideas [19] also led to the equivalence of injectivity with approximate finite dimensionality (also called hyperfiniteness) and also with the weak* CPAP (also called semidiscreteness). Since we have no analogue of approximate finite dimensionality (see however Remark 1.17), for which we refer to chapter XVI in [58, or to [4, chap. 11], we will concentrate on the parallel between weak* $\mathrm{CPAP} \Leftrightarrow$ injective and weak* $\mathrm{PAP} \Leftrightarrow$ seemingly injective. We will focus on a result due to Effros and Lance from [23, Prop. 4.5], with the refinement (i) $\Leftrightarrow$ (ii) proposed in [14].

Theorem 6.1 ([23]). The following properties of a von Neumann algebra $M \subset B(H)$ are equivalent:

(i) $M$ has the weak* CPAP (i.e. is semidiscrete according to [23]).

(ii) There is a net of integers $n(\alpha)$ and normal finite rank maps $T_{\alpha}: M \rightarrow M$ of the form

$$
M \stackrel{u_{\alpha}}{\longrightarrow} M_{n(\alpha)} \stackrel{v_{\alpha}}{\longrightarrow} M
$$

such that $u_{\alpha}, v_{\alpha}$ are both unital and c.p. (so that $\left.\left\|u_{\alpha}\right\| \leq 1,\left\|v_{\alpha}\right\| \leq 1\right), u_{\alpha}$ is normal and $T_{\alpha}(x)=$ $v_{\alpha} u_{\alpha}(x) \rightarrow x$ weak $^{*}$ for any $x \in M$.

(iii) The $*$-homomorphism $p: M^{\prime} \otimes M \rightarrow B(H)$ defined by $p(y \otimes x)=y x$ extends to a contractive *-homomorphism on $M^{\prime} \otimes_{\min } M$.

Since the weak* CPAP does not depend on the embedding $M \subset B(H)$, one may choose a convenient embedding in (iii). If $M \subset B(H)$ is in standard form then $M^{\prime} \simeq \bar{M}$. In that case, $p$ becomes the map taking $\bar{y} \otimes x$ to $J y J x$, or equivalently it becomes the representation defined on 
$\bar{M} \otimes_{\min } M$ by $\rho\left(\sum \overline{y_{j}} \otimes x_{j}\right)=\sum J y_{j} J x_{j}$ (see $\oint 3$ for the notation). Then the preceding statement says that $M$ has the weak* CPAP if and only if for any $t=\sum \overline{y_{j}} \otimes x_{j} \in \bar{M} \otimes M$ we have

$$
\|\rho(t)\|_{B(H)} \leq\|t\|_{\bar{M} \otimes_{\min } M}
$$

Assume $M \sigma$-finite for simplicity and let $\xi \in P^{\natural}$ be a unit vector that is both separating and cyclic. We claim that it suffices to have for any $t \in \bar{M} \otimes M$

$$
|\langle\xi, \rho(t) \xi\rangle| \leq\|t\|_{\bar{M} \otimes_{\min } M}
$$

Indeed, if this holds then $F$ defined by $F(t)=\langle\xi, \rho(t) \xi\rangle$ defines a (vector) state on $\bar{M} \otimes_{\min } M$, and $\xi$ is a cyclic vector for $\rho$. Thus the GNS representation of $F$ on $\bar{M} \otimes_{\min } M$ (which is clearly contractive) can be identified with $\rho$. So we recover (6.1). Thus (6.1) $\Leftrightarrow(6.2)$.

By a combination of results due to Haagerup (unpublished) for any finite set $\left(x_{j}\right)$ in $M$ we have

$$
\left\|\sum \overline{x_{j}} \otimes x_{j}\right\|_{\max }=\left\|\rho\left(\sum \overline{x_{j}} \otimes x_{j}\right)\right\|_{B(H)} .
$$

See [52, Th. 23.39] for a detailed proof. In the semifinite case (6.3) essentially goes back to [49].

The identity (6.3) shows that (6.1) holds if and only if for any finite set $\left(x_{j}\right)$ in $M$ we have

$$
\left\|\sum \overline{x_{j}} \otimes x_{j}\right\|_{\bar{M} \otimes_{\max } M}=\left\|\sum \overline{x_{j}} \otimes x_{j}\right\|_{\bar{M} \otimes_{\min } M} .
$$

This fact a posteriori clarifies the equivalence between the weak* CPAP and (6.1), which boils down to (i) $\Leftrightarrow$ (iii) in Theorem 6.1, This was proved by Effros and Lance in [23] which circulated (in preprint form) before Connes and Choi-Effros proved in [19, 16] its equivalence with injectivity. At the time Effros and Lance proved that the weak* CPAP implies injectivity but could not prove the converse. After the Connes paper [19] it could be proved that injectivity implies (iii) in Theorem 6.1 (see [62]), and that gave the desired converse.

Theorem 6.2. Let $M$ be a von Neumann algebra. The following are equivalent, where in (iii) we assume $M \sigma$-finite in standard form, and in (iii)' we assume $M$ finite with a faithful, normal and normalized trace $\tau$.

(i) $M$ has the matricial weak* CPAP.

(ii) There is a unital c.p. normal injective linear map $u: M \rightarrow B(H)$ such that $u_{\mid u(M)}^{-1}: u(M) \rightarrow M$ is positive and for any finite set $\left(x_{j}\right)$ in $M$ we have

$$
\left\|\sum \overline{x_{j}} \otimes x_{j}\right\|_{\bar{M} \otimes_{\max } M} \leq\left\|\sum \overline{u\left(x_{j}\right)} \otimes u\left(x_{j}\right)\right\|_{\overline{B(H)} \otimes_{\min } B(H)} .
$$

(iii) There is a faithful normal state $\varphi$ with associated unit vector $\xi \in P^{\natural}$ such that

$$
\left|\sum\left\langle\xi, J x_{j} J x_{j} \xi\right\rangle\right| \leq \sup \left\|\sum \overline{u\left(x_{j}\right)} \otimes u\left(x_{j}\right)\right\|_{\overline{B(H)} \otimes_{\min } B(H)}
$$

where the sup runs over all $H$ and all unital c.p. normal maps $u: M \rightarrow B(H)$.

(iii)' For any finite set $\left(x_{j}\right)$ in $M$ we have

$$
\sum \tau\left(x_{j}{ }^{*} x_{j}\right) \leq \sup \left\|\sum \overline{u\left(x_{j}\right)} \otimes u\left(x_{j}\right)\right\|_{\overline{B(H)} \otimes_{\min } B(H)},
$$

where the sup runs over all $H$ and all normal $*$-homomorphisms $u: M \rightarrow B(H)$. 
This statement can be proved exactly in the same way as for Theorem 6.4 below.

Remark 6.3. By what precedes for $M$ finite equipped with $\tau$ : (6.2) holds if and only if for any finite set $\left(x_{j}\right)$ in $M$ we have

$$
\tau\left(\sum x_{j}{ }^{*} x_{j}\right) \leq\left\|\sum \overline{x_{j}} \otimes x_{j}\right\|_{\bar{M} \otimes_{\min } M} .
$$

We would like to indicate how this was originally derived from Connes's ideas. The usual representation by left multiplication of $M$ on $L_{2}(\tau)$ is a standard form for $M$. Assuming $\tau(1)=1$, we may take $\xi=1$. Then (6.2) holds if and only if for any finite sets $\left(x_{j}\right),\left(y_{j}\right)$ in $M$ we have

$$
\left|\tau\left(\sum y_{j}^{*} x_{j}\right)\right| \leq\left\|\sum \overline{y_{j}} \otimes x_{j}\right\|_{\bar{M} \otimes_{\min } M} .
$$

In [19], Connes observed that if $M$ is a finite factor then for (6.2) to hold it suffices that it holds for $x_{j}$ unitary and $x_{j}=y_{j}$. The route he used went through the equivalence with injectivity. The condition becomes $n \leq\left\|\sum_{1}^{n} \overline{x_{j}} \otimes x_{j}\right\|_{\text {min }}$ for all finite sets of unitaries $\left(x_{j}\right)$, and now of course the converse inequality trivially holds. By a reasoning used commonly for means on amenable groups, this leads to a net $\left(h_{\alpha}\right)$ of Hilbert-Schmidt operators with $\left\|h_{\alpha}\right\|_{2}^{2}=\operatorname{tr}\left|h_{\alpha}\right|^{2}=1$ such that $\left\|h_{\alpha}-x h_{\alpha} x^{*}\right\|_{2} \rightarrow 0$ for all unitary $x$. Then Connes introduced the functional $\Phi$ defined on $B(H)$ by $\Phi(T)=\lim _{\mathcal{U}} \operatorname{tr}\left(h_{\alpha}^{*} T h_{\alpha}\right)$ (that he called a "hypertrace") from which injectivity can be derived. By the same route, the restriction to factors was removed in [29, Lemma 2.2] by Haagerup who proved that it suffices for (6.2) to hold for all $\left(x_{j}\right)$ of the form $\left(U_{j} q\right)$ with $\left(U_{j}\right)$ unitaries and $q \neq 0$ an arbitrary central projection, as follows

$$
n \leq\left\|\sum \overline{U_{j} q} \otimes U_{j} q\right\|_{\bar{M} \otimes_{\min } M} .
$$

It is easy to deduce from this the following claim (for $M$ finite equipped with $\tau$ ):

(6.2) holds if and only if for any finite set $\left(x_{j}\right)$ in $M$ we have

$$
\tau\left(\sum x_{j}{ }^{*} x_{j}\right) \leq\left\|\sum \overline{x_{j}} \otimes x_{j}\right\|_{\bar{M} \otimes_{\min } M} .
$$

Indeed, if this holds then we must have $\tau(q) n \leq\left\|\sum \overline{U_{j} q} \otimes U_{j} q\right\|_{\bar{M} \otimes_{\min } M}$, but then applying the same to $\left(\sum \overline{U_{j} q} \otimes U_{j} q\right)^{m}$ we get $\tau(q) n^{m} \leq\left\|\sum \overline{U_{j} q} \otimes U_{j} q\right\| \frac{m}{M \otimes_{\min } M}$, and after taking the $m$-th root and letting $m \rightarrow \infty$ we recover (6.6).

The analogue of the preceding statement for the PAP in place of the CPAP is as follows:

Theorem 6.4. Let $M$ be a von Neumann algebra. The following are equivalent, where in (iii) we assume $M \sigma$-finite in standard form, and in (iii)' we assume $M$ finite with $\tau$ as before.

(i) $M$ has the matricial weak* PAP.

(ii) There is a unital positive normal injective linear map $u: M \rightarrow B(H)$ such that $u_{\mid u(M)}^{-1}: u(M) \rightarrow$ $M$ is positive and for any finite set $\left(x_{j}\right)$ in $M$ we have

$$
\left\|\sum \overline{x_{j}} \otimes x_{j}\right\|_{\bar{M} \otimes_{\max } M} \leq\left\|\sum \overline{u\left(x_{j}\right)} \otimes u\left(x_{j}\right)\right\|_{\overline{B(H)} \otimes_{\min } B(H)} .
$$

(iii) There is a faithful normal state $\varphi$ with associated unit vector $\xi \in P^{\natural}$ such that

$$
\left|\sum\left\langle\xi, J x_{j} J x_{j} \xi\right\rangle\right| \leq \sup \left\|\sum \overline{u\left(x_{j}\right)} \otimes u\left(x_{j}\right)\right\|_{\overline{B(H)} \otimes_{\min } B(H)}
$$

where the sup runs over all $H$ and all unital positive normal maps $u: M \rightarrow B(H)$. 
(iii)' For any finite set $\left(x_{j}\right)$ in $M$ we have

$$
\sum \tau\left(x_{j}{ }^{*} x_{j}\right) \leq \sup \left\|\sum \overline{u\left(x_{j}\right)} \otimes u\left(x_{j}\right)\right\|_{\overline{B(H)} \otimes_{\min } B(H)},
$$

where the sup runs over all $H$ and all unital positive normal maps $u: M \rightarrow B(H)$.

Proof. Assume (i). By Theorem 1.11, $M$ is seemingly injective. Let $u, v$ be as in (1.1). Then $v u\left(x_{j}\right)=x_{j}$ for any $x_{j} \in M$. Therefore, since $v$ is here c.p. (4.5) (with $u\left(x_{j}\right)$ in place of $x_{j}$ ) implies (ii). (ii) $\Rightarrow$ (iii) is clear by Remark 3.3. Assume (iii). If we consider the single embedding $x \mapsto \oplus u(x)$ where the direct sum runs over all possible normal unital positive $u: M \rightarrow B(H)$ (say with some bound on the cardinality of $H$ ) then we see that (iii) holds for a single $u$. Furthermore, repeating that $u$ infinitely many times, we may assume that (iii) holds for some embedding $u$ with infinite multiplicity. By the argument used in Proposition 4.1 we have the situation described in (5.12). The proof of Theorem 1.11 shows that $M$ has the matricial weak* PAP, and hence (i) holds. In the finite case, the proof of Theorem 1.11 shows that (iii)' $\Rightarrow$ (i) and (ii) $\Rightarrow$ (iii)' is clear by Remark 3.3 .

Remark 6.5. In sharp contrast with the c.p. case, we do not know whether the weak* PAP implies the matricial weak* PAP, or equivalently whether the weak* PAP implies QWEP.

\section{Counterexamples}

In [56] Szankowski proved that $B(H)$ fails the AP in Grothendieck's sense. A fortiori, $B(H)^{*}$ fails MAP and hence $B(H)^{* *}$ certainly fails the weak* PAP and hence is not seemingly injective. Similarly $\mathbb{B}=\left(\oplus \sum M_{n}\right)_{\infty}$ is not seemingly injective.

We can derive from this the existence of finite factors (unfortunately not really too "concrete") that are not seemingly injective:

Proposition 7.1. Let $\omega$ be a free ultrafilter on $\mathbb{N}$. The ultraproduct $\prod M_{n} / \omega$ and the ultrapower $R^{\omega}$ of the hyperfinite factor $R$ are not seemingly injective.

Remark 7.2. Proposition 5.1 obviously remains valid if $\left\{B\left(H_{\alpha}\right)\right\}$ is replaced by a family $\left\{M_{\alpha}\right\}$ of seemingly injective von Neumann algebras.

Remark 7.3. This shows in particular that if $\mathcal{M}$ is seemingly injective then any von Neumann subalgebra $M \subset \mathcal{M}$ admitting a unital positive (surjective) projection $P: \mathcal{M} \rightarrow M$ is also seemingly injective. Note that when $\mathcal{M}$ is finite there is automatically a conditional expectation and hence a u.c.p. projection $P: \mathcal{M} \rightarrow M$.

Proof of Proposition 7.1. Assume for contradiction that if either $\mathcal{M}=R^{\omega}$ or $\mathcal{M}=\prod M_{n} / \omega$, the algebra $\mathcal{M}$ is seemingly injective. We will show that this implies that any QWEP von Neumann algebra $M$ is seemingly injective.

Any QWEP (or "Connes embeddable") finite $M$ with separable predual embeds in $\mathcal{M}$ and hence is seemingly injective by Remark [7.3. When $M$ is finite with $M_{*}$ non separable, this remains true if $M$ is $\sigma$-finite, or equivalently admits a faithful normal tracial state. Indeed, we may then view $M$ as the directed union of a family of finitely generated subalgebras $\left\{M_{\alpha}\right\}$ (with conditional expectations $P_{\alpha}: M \rightarrow M_{\alpha}$ ). Each $M_{\alpha}$ has a separable predual. Then Remark 7.2 (or Proposition 5.1 applied to $I d_{M}$ ) shows that any $M$ which is QWEP, $\sigma$-finite and finite is seemingly injective. By Remark 7.2 again, the same remains true if finite is replaced by semifinite (indeed, we can apply Proposition 5.1 with $T=I d_{M}$ and with $\left\{B\left(H_{\alpha}\right)\right\}$ replaced by a family $\left\{M_{\alpha}\right\}$ of QWEP finite and 
$\sigma$-finite algebras of the form $M_{\alpha}=p_{\alpha} M p_{\alpha}$ where $p_{\alpha}$ are suitably chosen finite projections in $M$ ). By Takesaki's duality theorem (as described in [52, Th.11.3]), any $M$ embeds as a von Neuman subalgebra in some semifinite $\mathcal{M}$ in such a way that there is a c.p. contractive projection from $\mathcal{M}$ onto $M$, and moreover $\mathcal{M}$ embeds in $M$ as a von Neumann subalgebra in such a way that there is a u.c.p. projection from $M$ to $\mathcal{M}$. If $M$ is QWEP and $\sigma$-finite so is $\mathcal{M}$. (The fact that $\mathcal{M}$ inherits QWEP from $M$ follows e.g. from Remark 1.9]) Thus our inital assumption implies that any $\sigma$-finite (and QWEP) $M$ is seemingly injective. Now let $M$ be arbitrary (and QWEP). By a classical structural theorem (see [22, Ch. III §1 Lemma 7] (p.224 in the French edition and p. 291 in the English one) $M$ admits a decomposition as a direct sum

$$
M \simeq\left(\oplus \sum_{i \in I} B\left(\mathcal{H}_{i}\right) \bar{\otimes} N_{i}\right)_{\infty}
$$

where the $N_{i}$ 's are $\sigma$-finite (=countably decomposable), the $\mathcal{H}_{i}$ 's are Hilbert spaces and $\bar{\otimes}$ denotes the (von Neumann algebra sense) tensor product. Note that the $N_{i}$ 's inherit QWEP from $M$.

We claim that, under our assumption, any QWEP $M$ as in (7.1) is seemingly injective. Using Remark 7.2 as before we can reduce this to the case when $I$ is a countable (or even finite) set and the $\mathcal{H}_{i}$ 's are separable (or even finite dimensional). In the latter case $M$ is $\sigma$-finite, so the first part of the argument completes the proof of the claim.

Since we know (by [56]) that $M=B(H)^{* *}$ contradicts this, our initial assumption does not hold.

\section{Remote injectivity}

We remind the reader that $M$ is called remotely injective if we have a factorization as in (1.1) but where we only assume $u$ normal and isometric, and $v$ completely contractive. Let us first observe that this implies that $M$ is QWEP. In fact it suffices for this to assume that $u, v$ are both contractions. Indeed, this implies that $M^{*}$ embeds isometrically into $B(H)^{*}$, and hence that $M_{*}$ is finitely representable in $B(H)_{*}$ and this implies by Kirchberg's results (see [52, §15]) that $M$ is QWEP.

The next statement gives a nice sounding reformulation of remote injectivity in terms of maximal operator spaces. The latter were introduced by Blecher and Paulsen [8] (see e.g. [51, §3] for more on this). Given a Banach space $X$ the operator space $\max (X)$ is characterized by the property that it is isometrically isomorphic to $X$ and for any $\operatorname{map} u: \max (X) \rightarrow B(H)$ we have $\|u\|_{c b}=\|u\|$. One way to produce a completely isometric realization of $\max (X)$ is like this: let $\mathcal{C}$ denote the collection of all contractions $v: X \rightarrow B\left(H_{v}\right)$ (with $H_{v}$ either of cardinality at most that of $X$ or simply finite dimensional) and then consider the embedding

$$
J: X \rightarrow B\left(\oplus_{v \in \mathcal{C}} H_{v}\right) \text { defined by } x \mapsto \oplus_{v \in \mathcal{C}} v(x) .
$$

Then $\max (X)$ can be identified with $J(X) \subset B\left(\oplus_{v \in \mathcal{C}} H_{v}\right)$.

Proposition 8.1. A von Neumann algebra $M$ is remotely injective if and only if the natural complete contraction (defined by the identity map on $M$ )

$$
\Phi: \max (M) \rightarrow M
$$

factors completely contractively through $B(H)$ for some $H$.

Proof. Assume there are $u, v$ such that $\Phi=v u$ with $\|u: \max (M) \rightarrow B(H)\|_{c b}=\|u\|=1$ and $\|v\|_{c b}=1$. Since $M$ is a dual space (i.e. $M=\left(M_{*}\right)^{*}$ ) the o.s. $\max (M)$ is a dual o.s. (it is the 
dual of the o.s. $\min \left(M_{*}\right)$ in the sense of [8]). It follows (see e.g. [7, Lemma 1.4 .7 p. 23]) that there is a completely isometric embedding $U: \max (M) \rightarrow B(\mathcal{H})$ realizing $\max (M)$ as a weak*closed subspace. Let $X=U(\max (M))$ be the range of $U$. Then $M$ is isometric to $X$ and by Sakai's uniqueness of predual theorem $U$ is normal. By the injectivity of $B(H)$ there is a map $\widetilde{u}: B(\mathcal{H}) \rightarrow B(H)$ with $\|\widetilde{u}\|_{c b}=\|u\|_{c b}$ extending $u$. Now if we let $V=v \widetilde{u}: B(\mathcal{H}) \rightarrow M$ we obtain $I d_{M}=V U$ with $V, U$ satisfying the conditions required to make $M$ remotely injective. This proves the if part. The converse is obvious.

Ozawa connected in [43] and [44] the lifting problems described in Remark 2.2 with maximal operator spaces in the sense of Blecher and Paulsen [8] and local reflexivity in the sense of Effros and Haagerup (see [24] or [51, §18]). Within operator spaces, it is convenient to introduce constants relative to these properties, as follows.

Definition 8.2. An operator space (o.s. in short) $X \subset B(H)$ is called $\lambda$-maximal if any bounded linear map $u: X \rightarrow Y$ into an arbitrary operator space $Y$ is completely bounded and satisfies $\|u\|_{c b} \leq \lambda\|u\|$.

For example for any Banach space $X$ the o.s. $\max (X)$ described above is 1-maximal.

Definition 8.3. An o.s. $X \subset B(H)$ is called $\lambda$-locally reflexive if for any finite dimensional (f.d. in short) subspace $E \subset X^{* *}$ there is a net of maps $u_{\alpha}: E \rightarrow X$ with $\left\|u_{\alpha}\right\|_{c b} \leq \lambda$ that tend pointwise-weak* to the inclusion $E \subset X^{* *}$.

To make the link with Ozawa's questions, we will use the following simple lemma. Whether an analogous lemma is valid with $Y$ finite dimensional (possibly in some variant involving factoring the inclusion $E \subset X$ through $Y$ ) is the central open question discussed by Oikhberg in [41]. It is closely linked to Ozawa's question whether all maximal spaces are locally reflexive.

Lemma 8.4. Let $E \subset X$ be a f.d. (or merely separable) subspace of a maximal o.s. There is a separable maximal subspace $Y$, such that $E \subset Y \subset X$.

Proof. This is stated as Lemma 3.4 in [44]. For a detailed proof see Exercise 3.8 in [51, p. 80], with solution in [51, p. 429]. The latter is based on Paulsen's description of the unit ball of $M_{n}(X)$ when $X$ is maximal (see [46] or e.g. [52, p. 72]). This lemma can also be checked by a routine duality argument.

An o.s. $X$ has the OLLP if any complete contraction $u: X \rightarrow C / \mathcal{I}$ into an arbitrary quotient $C^{*}$-algebra is locally liftable in the following sense: for any f.d. subspace $E \subset X$ there is a complete contraction $u^{E}: E \rightarrow C$ that lifts $u_{\mid E}$.

Let us say that an o.s. $X$ is strongly $\lambda$-maximal if $X$ is the union of an increasing net of f.d. $\lambda$-maximal subspaces $E_{i}$ such that $X=\overline{\cup E_{i}}$. In [43] and [4] Ozawa observed that any strongly 1-maximal $X$ has the OLLP. Motivated by this observation, he asked whether any 1-maximal o.s. has the OLLP. By Lemma 8.4 the question reduces immediately to the case when $X$ is separable. We think that the answer is negative and that there are 1-maximal o.s. that are not strongly $\lambda$-maximal for any $1 \leq \lambda<\infty$. In fact the space $X=\max \left(B\left(\ell_{2}\right)^{* *}\right)$ is a natural candidate for a counterexample. This question is related to our main topic via the following.

Proposition 8.5. Let $M$ be a $Q W E P$ von Neumann algebra. If $\max (M)$ has the OLLP then $M$ is remotely injective. 
Proof. As explained in the proof of Proposition 8.1 we may assume that we have a normal isometric embeding $U: M \rightarrow B(\mathcal{H})$ realizing $\max (M)$ as a weak* closed subspace of $B(\mathcal{H})$. For simplicity we identify $\max (M)$ with $U(M)$. Let $W, q, w_{1}, w_{2}$ be as in Lemma 5.4. Let $u=\Phi: \max (M) \rightarrow M$. Let $E \subset \max (M)$ be f.d. By the OLLP there is $u^{E}: E \rightarrow W$ with $\left\|u^{E}\right\|_{c b} \leq 1$ such that $q u^{E}=u_{\mid E}$. By the injectivity of $B(H)$ the map $w_{1} u^{E}: E \rightarrow B(H)$ admits an extension $v^{E}: B(\mathcal{H}) \rightarrow B(H)$ with $\left\|v^{E}\right\|_{c b} \leq 1$. Then the map $w^{E}=w_{2} v^{E}: B(\mathcal{H}) \rightarrow M$ is such that $w^{E}(x)=x$ for any $x \in E$ and $\left\|w^{E}\right\|_{c b} \leq 1$. Let $\mathcal{U}$ be an ultrafilter refining the net of finite dimensional subpaces $\{E \mid E \subset M\}$. We define $V: B(\mathcal{H}) \rightarrow M$ by

$$
\forall b \in B(\mathcal{H}) \quad V(b)=\text { weak }^{*} \lim _{\mathcal{U}} w^{E}(b) .
$$

Then $V(b) \in M, V(x)=x$ for any $x \in M$, so that $I d_{M}=V U$ and $\|V\|_{c b} \leq 1$.

Let us say that a surjective $*$-homomorphism $q: W \rightarrow M$ (here between $C^{*}$-algebras) admits contractive (resp. bounded) liftings if for any separable Banach space $Y$ and any contractive map $u: Y \rightarrow M$ there is a contractive (resp. bounded) map $\hat{u}: Y \rightarrow W$ that lifts $u$, meaning that $q \hat{u}=u$. As discussed already in Remark 2.2 there are no known examples of surjective $*$-homomorphism $q: W \rightarrow M$ that fail this. This motivates the next statement.

Proposition 8.6. Let $M$ be a $Q W E P$ von Neumann algebra. Let $W$ be a WEP $C^{*}$-algebra and let $q: W \rightarrow M$ be a surjective $*$-homomorphism. If $q$ admits contractive liftings then $M$ is remotely injective.

Proof. Let $U: M \rightarrow B(\mathcal{H})$ be as in the preceding proof. We again identify $\max (M)$ with $U(M)$. By Lemma 8.4, for any f.d. $E \subset \max (M)$ there is a separable $Y \subset \max (M)$ such that $E \subset Y$ that is a maximal o.s. i.e. we have $\max (Y)=Y$. Let $u: Y \rightarrow M$ be the inclusion map of $Y$ into $M$. By our assumption, $u$ admits a lifting $\hat{u}: Y \rightarrow W$ with $\|\hat{u}\| \leq 1$. Then $\|\hat{u}\|_{c b}=1$ by maximality of $Y$. By the injectivity of $B(H)$, the map $w_{1} \hat{u}$ admits an extension $w_{3}: B(\mathcal{H}) \rightarrow B(H)$ with $\left\|w_{3}\right\|_{c b}=1$. Let $w^{E}=w_{2} w_{3}: B(\mathcal{H}) \rightarrow M$. Note $w^{E}(x)=u(x)=x$ for any $x \in Y$, and a fortiori for any $x \in E$. At this point we can conclude as in the preceding proof.

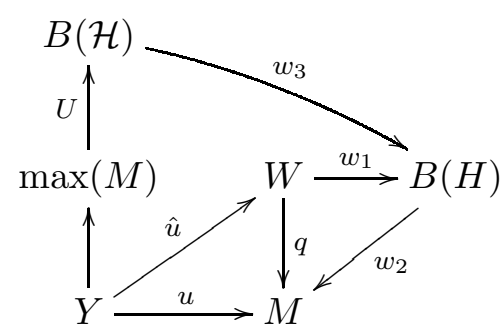

The same argument shows that if $q$ admits bounded liftings then the isometric embedding $M \subset B(\mathcal{H})$ is complemented, i.e. there is a bounded projection $P: B(\mathcal{H}) \rightarrow M$.

Remark 8.7. We failed to prove an analogue of Proposition 8.6 for positive contractive liftings to conclude that $M$ is seemingly injective. We suspect the description in [48] of the analogue of $\max (X)$ when $X$ is an operator system should be useful, but the analogue of the normal embedding $U$ seems to be missing.

Remark 8.8. Let $M$ be a QWEP $C^{*}$-algebra. Then there is a normal embedding $M^{* *} \subset B(H)^{* *}$ with a unital completely positive projection from $B(H)^{* *}$ onto $M^{* *}$ (see [52, p. 207]), and the projection can be chosen normal by [52, p. 148]. If $M$ is a von Neumann algebra there is a normal 
(non-unital) embedding $M \subset M^{* *}$ (this is not the canonical one) with a unital completely positive normal projection from $M^{* *}$ onto $M$. Therefore we have a factorization

$$
I d_{M}: M \stackrel{U}{\longrightarrow} B(H)^{* *} \stackrel{V}{\longrightarrow} M
$$

where $U, V$ are normal unital c.p. maps. Thus, if $B(H)^{* *}$ was remotely (resp. seemingly) injective, the same would be true for any QWEP $M$.

The next lemma is a well known duality argument (see e.g. [52, p. 440]). We denote there by $S_{1}^{n}$ the o.s. dual of $M_{n}$ that is determined by the isometric identity $C B\left(S_{1}^{n}, E\right)=M_{n}(E)$ for any o.s. $E$ (see [24, 51, 47]).

Lemma 8.9 (Hahn-Banach argument). Let $X \subset B$ be a completely isometric inclusion of o.s. Let $T: X \rightarrow C^{*}$ be a map into the dual of another o.s. $C$. The following are equivalent:

(i) There is an extension $\widetilde{T}: B \rightarrow C^{*}$ with $\|\widetilde{T}\|_{c b} \leq \lambda$.

(ii) For any $n$, any $S \subset S_{1}^{n}$ and any $u: S \rightarrow X$ that admits an extension $\hat{u}: S_{1}^{n} \rightarrow B$ with $\|\hat{u}\|_{c b}=1$ there is a map $\widetilde{u}: S_{1}^{n} \rightarrow C^{*}$ such that $\widetilde{u}_{\mid S}=T u$ with $\|\widetilde{u}\|_{c b} \leq \lambda$.

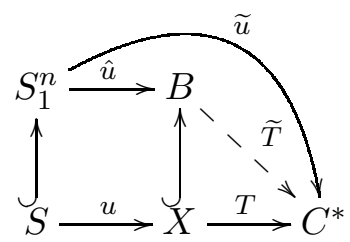

Note that if $B$ is injective (e.g. when $B=B(H)$ ) then (ii) is the same as

(ii)' For any $n$, any $S \subset S_{1}^{n}$ and any $u: S \rightarrow X$ with $\|u\|_{c b}=1$ there is a map $\tilde{u}: S_{1}^{n} \rightarrow C^{*}$ such that $\widetilde{u}_{\mid S}=T u$ with $\|\widetilde{u}\|_{c b} \leq \lambda$.

Theorem 8.10. Let $A$ be any unital $C^{*}$-algebra. If $\max (A)$ is locally reflexive (with constant 1 ) and $A$ WEP then $A^{* *}$ is remotely injective.

Proof. Let $U: \max \left(A^{* *}\right) \rightarrow B(\mathcal{H})$ be a completely isometric embedding as a weak*-closed subspace. Let $\Phi: \max \left(A^{* *}\right) \rightarrow A^{* *}$ be as before the complete contraction defined by the identity on $A^{* *}$. We claim that if $\max (A)$ is $\lambda$-locally reflexive we have a factorization of $\Phi: \max \left(A^{* *}\right) \rightarrow A^{* *}$ of the form $\Phi=v U$ for some completely contractive $v: B(\mathcal{H}) \rightarrow A^{* *}$. To check that we use Lemma 8.9 and the local reflexivity. By (ii)' in Lemma 8.9 it suffices to prove that for any $S \subset S_{1}^{n}$ and any $u: S \rightarrow \max \left(A^{* *}\right)$ with $\|u\|_{c b}=1$ there is a map $\widetilde{u}: S_{1}^{n} \rightarrow A^{* *}$ with $\|\widetilde{u}\|_{c b} \leq \lambda$ that extends $u$ in the sense that $\widetilde{u}_{\mid S}=\Phi u$. Now since $\max (A)^{* *}=\max \left(A^{* *}\right)$ the LR assumption on $\max (A)$ implies that there is a net of c.c. maps $u_{i}: S \rightarrow \max (A)$ tending pointwise weak* to $u$. Now $\Phi u_{i}: S \rightarrow A$ is a complete contraction, and since $A$ has the WEP we have c.c. maps $v_{i}: S_{1}^{n} \rightarrow A$ extending $\Phi u_{i}$. We now use an ultrafilter $\mathcal{U}$ and define $\widetilde{u}(x)=\lim _{\mathcal{U}} v_{i}(x) \in A^{* *}$ for any $x \in S_{1}^{n}$, the limit being in the $\sigma\left(A^{* *}, A^{*}\right)$-sense. Now $\widetilde{u}: S_{1}^{n} \rightarrow A^{* *}$ is our extension since for any $s \in S$ we have $\widetilde{u}(s)=\lim _{\mathcal{U}} v_{i}(s)=\lim _{\mathcal{U}} \Phi u_{i}(s)=\Phi u(s)$ for limits in the same sense. Thus $\widetilde{u}_{\mid S}=\Phi u$.

Corollary 8.11. If $\max (B(H))$ is 1-locally reflexive then $B(H)^{* *}$ is remotely injective.

Proof. Indeed, $B(H)$ is injective and hence has the WEP. 


\section{Some questions}

By a well known result (see [14, 14]) a $C^{*}$-algebra is nuclear if and only if $A^{* *}$ is injective. One could name "seemingly nuclear" the $C^{*}$-algebras $A$ for which $A^{* *}$ is seemingly injective. Unfortunately at this stage we do not have any valuable information on this notion.

Similarly, a discrete group $G$ could be called "seemingly amenable" if $M=L(G)$ is "seemingly injective". By Remark 1.13 this would lead to the shocking assertion that free groups are seemingly amenable!

At the moment we do not have any example of a group that is not seemingly amenable but we suspect that such examples exist, for instance among property $(\mathrm{T})$ groups (for which we refer the reader to [6]).

As for von Neumann algebras, it would be nice to exhibit more examples of $M$ 's that are QWEP but not seemingly injective, without relying on Szankowski's construction [56].

Another puzzling question is whether it is really essential that the map $u$ in Definition [1.1 should be normal. It seems essential for the proof of Theorem 1.11. So we ask: does any QWEP von Neumann algebra $M$ admit a factorization as in (1.1) with $u$ isometric and $\|v\|_{c b}=1$ ? Is any non-nuclear QWEP von Neumann algebra $M$ isomorphic as a Banach space to $B(H)$ for some $H$ ?

Unfortunately, we could not decide the following (which were our original motivation):

\section{Conjectures:}

(i) Any remotely injective von Neumann algebra $M$ has the (Banach space sense) weak* MAP (equivalently $M_{*}$ has the MAP).

(ii) $B(H)^{* *}$ (say for $H=\ell_{2}$ ) is not remotely injective.

(ii)' There is a QWEP von Neumann algebra that is not remotely injective.

(iii) There exists an ideal in a separable $C^{*}$-algebra with no contractive lifting, and hence with lifting constant $>1$.

Note that by the same argument as for Proposition 7.1 (ii) implies that $R^{\omega}$ or $\prod M_{n} / \omega$ are not remotely injective. We feel that (ii) and (iii) should be true, but are less convinced about (i), due to multiple unsuccessful attempts (our initial hope was to prove that remotely injective implies seemingly injective).

We will show (i) $\Rightarrow$ (ii) $\Leftrightarrow$ (ii)' $\Rightarrow$ (iii). Obviously (i) implies (ii) by Szankowski's results on the failure of the AP for $B(H)$ or a fortiori for any non-nuclear von Neumann algebra. Indeed, his results imply that $B(H)^{*}$ fails the MAP, and hence that $B(H)^{* *}$ fails the weak* MAP. (ii) $\Rightarrow$ (ii)' is trivial since $B(H)^{* *}$ is QWEP (see [52, p. 205]). In fact (ii) and (ii)' are actually equivalent by Remark 8.8 .

The fact that (ii) implies (iii) follows from Proposition 8.6.

This argument shows that if (iii) fails then any QWEP $M$ is remotely injective, or equivalently (ii) fails.

In conclusion, if there exists a QWEP von Neumann algebra $M$ that is not remotely injective (which boils down to $M=B(H)^{* *}$ ), then there exists an ideal in a separable $C^{*}$-algebra with no contractive lifting, and $\max (B(H))$ is not locally reflexive (with constant 1). This would answer (at least for the constant 1 case) some of the questions raised in [43] and [44, and also in [41].

Lastly, we should mention that according to the recent very long preprint 35] there are von Neumann algebras that are not QWEP.

Acknowledgement: Thanks are due to the referees for useful comments and corrections. 


\section{References}

[1] C. Anantharaman and S. Popa, An introduction to II $I_{1}$-factors, Cambridge Univ. Press, to appear.

[2] T. B. Andersen, Linear extensions, projections, and split faces. J. Functional Analysis 17 (1974), 161-173.

[3] T. Ando, Closed range theorems for convex sets and linear liftings. Pacific J. Math. 44 (1973), 393-410.

[4] T. Ando, A theorem on nonempty intersection of convex sets and its application. $J$. Approximation Theory 13 (1975), 158-166.

[5] W. Arveson, Notes on extensions of $C^{*}$-algebras. Duke Math. J. 44 (1977), 329-355.

[6] B. Bekka, P. de la Harpe and A. Valette, Kazhdan's property (T), Cambridge University Press, Cambridge, 2008.

[7] D. P. Blecher and C. Le Merdy, Operator algebras and their modules: an operator space approach, Oxford Univ. Press, Oxford (2004).

[8] D. Blecher and V. Paulsen, Tensor products of operator spaces, J. Funct. Anal. 99 (1991), 262-292.

[9] G. Blower, The Banach space $B\left(\ell^{2}\right)$ is primary, Bull. London Math. Soc. 22 (1990), 176-182.

[10] N.P. Brown and N. Ozawa, $\mathrm{C}^{*}$-algebras and finite-dimensional approximations, Graduate Studies in Mathematics, 88, American Mathematical Society, Providence, RI, 2008.

[11] J. de Cannière and U. Haagerup, Multipliers of the Fourier algebras of some simple Lie groups and their discrete subgroups, Amer. J. Math. 107 (1985), 455-500.

[12] M.D. Choi, A Schwarz inequality for positive linear maps on $C^{*}$-algebras, Illinois J. Math. 18 (1974), 565-574.

[13] M.D. Choi and E. Effros, The completely positive lifting problem for $C^{*}$-algebras, Ann. of Math. 104 (1976), 585-609.

[14] M.D. Choi and E. Effros, Separable nuclear $C^{*}$-algebras and injectivity, Duke Math. J. 43 (1976), 309-322.

[15] M.D. Choi and E. Effros, Lifting problems and the cohomology of $C^{*}$-algebras. Canadian J. Math. 29 (1977), 1092-1111.

[16] M.D. Choi and E. Effros, Nuclear $\mathrm{C}^{*}$-algebras and injectivity: The general case, Indiana Univ. Math. J. 26 (1977), 443-446.

[17] M.D. Choi and E. Effros, Injectivity and operator spaces, J. Funct. Anal. 24 (1977), 156-209.

[18] E. Christensen and A. Sinclair, Completely bounded isomorphisms of injective von Neumann algebras, Proc. Edinburgh Math. Soc. (2) 32 (1989), 317-327. 
[19] A. Connes, Classification of injective factors. Cases $I I_{1}, I I_{\infty}, I I I_{\lambda}, \lambda \neq 1$, Ann. Math. (2) 104 (1976), 73-115.

[20] A. Connes and V. Jones, Property T for von Neumann algebras. Bull. London Math. Soc. 17 (1985), 57-62.

[21] K. Davidson. $C^{*}$-algebras by example, Fields Institute publication. Toronto, Amer. Math. Soc., Providence 1996.

[22] J. Dixmier, Les Algèbres d'Opérateurs dans l'Espace Hilbertien (Algèbres de von Neumann), Gauthier-Villars, Paris 1969. (in translation: von Neumann algebras, North-Holland, Amsterdam-New York 1981.)

[23] E. Effros and C. Lance, Tensor products of operator algebras, Adv. Math. 25 (1977), 1-34.

[24] E. Effros and Z.J. Ruan, Operator Spaces, Oxford Univ. Press, Oxford, 2000.

[25] E. Effros and E. Størmer, Positive projections and Jordan structure in operator algebras. Math. Scand. 45 (1979), 127-138.

[26] Y. Friedman and B. Russo, Solution of the contractive projection problem. J. Funct. Anal. 60(1985), 56-79.

[27] U. Haagerup, The standard form of von Neumann algebras, Math. Scand. 37 (1975), 271283.

[28] U. Haagerup, An example of a nonnuclear $C^{*}$-algebra, which has the metric approximation property, Invent. Math. 50 (1978/79), 279-293.

[29] U. Haagerup, Injectivity and decomposition of completely bounded maps, Springer Lecture Notes in Math. 1132 (1985), 170-222.

[30] U. Haagerup, Group $C^{*}$-algebras without the completely bounded approximation property, J. Lie Theory 26 (2016), 861-887.

[31] U. Haagerup and G. Pisier, Bounded linear operators between $C^{*}$-algebras, Duke Math. J. 71 (1993), 889-925.

[32] P. Harmand, D. Werner and W. Werner, M-ideals in Banach spaces and Banach algebras, Lecture Notes in Mathematics 1547 Springer-Verlag, Berlin, 1993.

[33] L.A. Harris, Bounded symmetric homogeneous domains in infinite dimensional spaces, Lecture Notes in Mathematics 364 Springer-Verlag, Berlin, 1974, pp. 13-40.

[34] L.A. Harris, A generalization of $C^{*}$-algebras, Proc. London Math. Soc. 42 (1981), 331-361.

[35] Z. Ji, A. Natarajan, T. Vidick, J. Wright, and H. Yuen, MIP* = RE, arxiv Jan. 2020.

[36] P. Jolissaint and A. Valette, Normes de Sobolev et convoluteurs bornés sur $L_{2}(G)$, Ann. Inst. Fourier (Grenoble) 41 (1991), 797-822.

[37] M. Junge and C. Le Merdy, Factorization through matrix spaces for finite rank operators between $C^{*}$-algebras, Duke Math. J. 100, (1999), 299-319. 
[38] A. Kavruk, Nuclearity related properties in operator systems. J. Operator Theory $\mathbf{7 1}$ (2014), 95-156.

[39] E. Kirchberg, On nonsemisplit extensions, tensor products and exactness of group $C^{*}$ algebras, Invent. Math. 112 (1993), 449-489.

[40] E. Kirchberg, Commutants of unitaries in UHF algebras and functorial properties of exactness, J. reine angew. Math. 452 (1994), 39-77.

[41] T. Oikhberg, Subspaces of maximal operator spaces, Integral Equations Operator Theory 48 (2004), 81-102.

[42] T. Oikhberg and H. P. Rosenthal, Extension properties for the space of compact operators, J. Funct. Anal. 179 (2001), 251-308.

[43] N. Ozawa, Local theory and local reflexivity for operator spaces, PhD thesis, 2001 (Texas A\& M University).

[44] N. Ozawa, On the lifting property for universal $C^{*}$-algebras of operator spaces, J. Operator Theory 46 (2001), no. 3, suppl., 579-591.

[45] N. Ozawa, About the QWEP conjecture, Internat. J. Math. 15 (2004), 501-530.

[46] V. Paulsen, The maximal operator space of a normed space. Proc. Edinburgh Math. Soc. 39 (1996) 309-323.

[47] V. Paulsen. Completely bounded maps and operator algebras, Cambridge Univ. Press, Cambridge, 2002.

[48] V. Paulsen, I. Todorov and M. Tomforde, Operator system structures on ordered spaces. Proc. Lond. Math. Soc. 102 (2011), 25-49.

[49] G. Pisier, Projections from a von Neumann algebra onto a subalgebra, Bull. Soc. Math. France 123 (1995), 139-153.

[50] G. Pisier, The operator Hilbert space $O H$, complex interpolation and tensor norms, Memoirs Amer. Math. Soc. 122 , 585 (1996), 1-103.

[51] G. Pisier, Introduction to operator space theory, Cambridge University Press, Cambridge, 2003.

[52] G. Pisier, Tensor products of $C^{*}$-algebras and operator spaces, The Connes-Kirchberg problem, Cambridge University Press, to appear.

Available at: https://www.math.tamu.edu/ pisier/TPCOS.pdf

[53] A. G. Robertson and R. Smith, Liftings and extensions of maps on $C^{*}$-algebras, J. Operator Theory 21 (1989), 117-131

[54] A. G. Robertson and S. Wassermann, Completely bounded isomorphisms of injective operator systems, Bull. London Math. Soc. 21 (1989), 285-290.

[55] S. Sakai, $C^{*}$-algebras and $W^{*}$-algebras, Springer-Verlag, New York-Heidelberg, 1971.

[56] A. Szankowski, $B(H)$ does not have the approximation property. Acta Math. 147 (1981), 89-108. 
[57] M. Takesaki, Theory of Operator algebras, vol. I. Springer-Verlag, Berlin, Heidelberg, New York, 1979.

[58] M. Takesaki, Theory of Operator algebras, vol. III. Springer-Verlag, Berlin, Heidelberg, New York, 2003.

[59] J. Tomiyama, On the projection of norm one in $W^{*}$-algebras. Proc. Japan Acad. 33 (1957), 608-612.

[60] J. Vesterstrøm, Positive linear extension operators for spaces of affine functions. Israel J. Math. 16 (1973), 203-211.

[61] S. Wassermann, On tensor products of certain group $C^{*}$-algebras, J. Funct. Anal. 23 (1976), 239-254.

[62] S. Wassermann, Injective $W^{*}$-algebras, Proc. Cambridge Phil. Soc. 82 (1977), 39-47. 


\title{
Corresponding author:
}

\author{
Gilles Pisier
}

Mathematics Dept.

Texas A\&M University

College Station, TX 77843-3368, USA

gilles.pisier@imj-prg.fr

Short statement on the research background and significance of the work:

This paper is devoted to a generalization of injectivity for von Neumann algebras. The surprising feature is that the generalized notion is satisfied by the von Neumann algebra of a non commutative free group which is the main fundamental example of a non-injective von Neumann algebra. The background involves von Neumann algebra theory as well as various notions of approximation properties of the identity by finite rank maps. 